Article

\title{
Straightforward Access to Enantioenriched cis-3-Fluoro-dihydroquinolin-4-ols Derivatives via Ru(II)-Catalyzed-Asymmetric Transfer Hydrogenation/Dynamic Kinetic Resolution
}

\author{
Ricardo Molina Betancourt, Phannarath Phansavath * and Virginie Ratovelomanana-Vidal *(D)
}

\section{check for} updates

Citation: Molina Betancourt, R.;

Phansavath, P.; Ratovelomanana-

Vidal, V. Straightforward Access to Enantioenriched cis-3-Fluorodihydroquinolin-4-ols Derivatives via $\mathrm{Ru}$ (II)-Catalyzed-Asymmetric Transfer Hydrogenation/Dynamic Kinetic Resolution. Molecules 2022, 27, 995. https://doi.org/10.3390/ molecules27030995

Academic Editor: Victor Mamane

Received: 10 January 2022

Accepted: 28 January 2022

Published: 1 February 2022

Publisher's Note: MDPI stays neutral with regard to jurisdictional claims in published maps and institutional affiliations.

Copyright: (c) 2022 by the authors. Licensee MDPI, Basel, Switzerland. This article is an open access article distributed under the terms and conditions of the Creative Commons Attribution (CC BY) license (https:// creativecommons.org/licenses/by/ $4.0 /)$.
CSB2D Team, CNRS, UMR 8060, Chimie ParisTech, Institute of Chemistry for Life and Health Sciences, PSL University, 75005 Paris, France; r.molina-betancourt@chimieparistech.psl.eu

* Correspondence: phannarath.phansavath@chimieparistech.psl.eu (P.P.); virginie.vidal@chimieparistech.psl.eu (V.R.-V.)

\begin{abstract}
Herein we report a practical method for the asymmetric transfer hydrogenation/dynamic kinetic resolution of $\mathrm{N}$-Boc 3-fluoro-dihydrotetrahydroquinolin-4-ones into the corresponding cisfluoro alcohols in 70-96\% yields, up to 99:1 diastereomeric ratio (dr) and up to $>99 \%$ ee (enantiomeric excess) by using the ruthenium complex Ts-DENEB and a formic acid/triethylamine (1:1) mixture as the hydrogen donor under mild conditions.
\end{abstract}

Keywords: asymmetric catalysis; reduction; fluorine; hydrogen transfer; ruthenium

\section{Introduction}

The number of bioactive molecules containing fluorine approved by the Food and Drug Administration (FDA) has greatly increased over time. The first decade of this century saw the introduction of 40 new compounds having a fluorine atom. This number represented at the time an important increase of $20 \%$ of the commercial drugs bearing at least one fluorine atom in their structure [1]. Compared to this, in 2018 alone, 17 fluorinecontaining pharmaceuticals were approved by the FDA [2]. In a single year, the number of new fluorinated drugs covered almost half of the increase of a whole decade.

This fluorine rush is easily explained by fluorine's ability to affect important parameters for a drug candidate, such as permeability or $\mathrm{pK}_{\mathrm{a}}$, and to modify its pharmacokinetics and pharmacodynamics. Fluorine is thus an element that gives control to medicinal chemists over tailoring the properties of a molecule. Organic chemists must then work in consideration of this increasing demand and develop new methodologies to introduce fluorine into complex molecules and into versatile building blocks [3].

The tetrahydroquinoline core is a very common scaffold found in several biologically active molecules and therapeutic agents [4]. Introducing a fluorine atom in its structure would bring a novelty into the medicinal chemist toolbox and, in theory, open up its use in the screening and development of new biologically active molecules. Asymmetric transfer hydrogenation (ATH) has been reported by Lassaletta and co-workers [5] as a method to access enantiomerically enriched fluoro-tetralol via a dynamic kinetic resolution (DKR) process (Scheme 1a). Following the premise of the fluorine need and the previous works in the laboratory including the ATH/DKR of 3-fluoro-chromanone derivatives [6-14] (Scheme 1b), we herein report an asymmetric transfer hydrogenation/dynamic kinetic resolution [15-24] (ATH/DKR) of tert-butyl 3-fluoro-4-oxo-3,4-dihydroquinoline-1(2H)carboxylates into the corresponding enantioenriched cis-fluorohydrins (Scheme 1c). 
Lassaletta and co-workers:<smiles>O=C1c2ccccc2CCC1F</smiles>

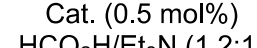

$\mathrm{HCO}_{2} \mathrm{H} / \mathrm{Et}_{3} \mathrm{~N}(1.2: 1)$

neat, r.t., $24 \mathrm{~h}$<smiles>O[C@H]1c2ccccc2CC[C@@H]1F</smiles>

1 example
$98 \%$ yield

$94 \%$ de

$97 \%$ ee (a)

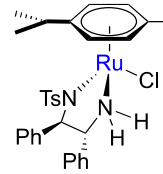

Cat.

)

Our previous work:<smiles>[X]CC(F)C(=O)C1=CC#[R]=CC=C1[X]</smiles>

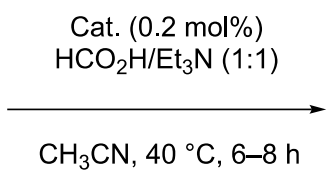

$\mathrm{CH}_{3} \mathrm{CN}, 40{ }^{\circ} \mathrm{C}, 6-8 \mathrm{~h}$

$\mathrm{R} \frac{1}{\mathrm{I}}$<smiles>[X]C[C@@H](F)[C@H](O)c1c[c-]ccc1[X]</smiles>

11 examples

$80-96 \%$ yield $92: 8$ to $99: 1 \mathrm{dr}$ $92->99 \%$ ee

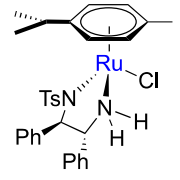

Cat.

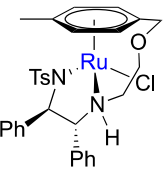

Cat.

Scheme 1. (a) ATH/DKR to access enantiomerically enriched fluoro-tetralol; (b) ATH/DKR of 3-fluoro-chromanone derivatives; (c) ATH/DKR of tert-butyl 3-fluoro-4-oxo-3,4-dihydroquinoline$1(2 H)$-carboxylates.

\section{Results and Discussion}

The study began with the synthesis of fluorinated dihydroquinolin-4-ones $\mathbf{2 a}-\mathbf{j}$ via an electrophilic fluorination of the $N$-Boc-protected heterocyclic ketones $\mathbf{1 a}-\mathbf{j}$ by using lithium bis(trimethylsilyl)amide as a base and NFSI as the fluorine source (Scheme 2).<smiles></smiles>

1

1a, $\mathrm{R}=\mathrm{H}$

$1 \mathrm{~b}, \mathrm{R}=6-\mathrm{Me}$

1c, $\mathrm{R}=6-\mathrm{OMe}$

1d, $\mathrm{R}=7-\mathrm{OMe}$

1e, $\mathrm{R}=6,7$-diOMe

\section{LiHMDS ( 1.05 equiv) $\mathrm{THF}, 0^{\circ} \mathrm{C}, 1.5 \mathrm{~h}$}

2. NFSI ( 1.2 equiv)

THF, $-78^{\circ} \mathrm{C} 30 \mathrm{~min}$

then r.t, $16 \mathrm{~h}$

1f, $R=6-F$

1g, $\mathrm{R}=6-\mathrm{CF}_{3}$

$1 \mathrm{~h}, \mathrm{R}=6-\mathrm{Cl}$

1i, $R=6-B r$

$1 j, R=6-I$<smiles>O=C1C2=C(C=C[R]C=C2)N(C(=O)OCc2ccccc2)CC1F</smiles>

$\begin{array}{ll}\mathbf{2 a}, 73 \% & \mathbf{2 f}, 86 \% \\ \mathbf{2 b}, 80 \% & \mathbf{2 g}, 45 \% \\ \mathbf{2 c}, 70 \% & \mathbf{2 h}, 39 \% \\ \mathbf{2 d}, 74 \% & \mathbf{2 i}, 41 \% \\ \mathbf{2 e}, 74 \% & \mathbf{2 j}, \mathbf{4 5 \%}\end{array}$

Scheme 2. Synthesis of fluorinated dihydroquinolin-4-ones $\mathbf{2 a - j}$.

3-Fluoro-dihydroquinolin-4-one carboxylate 2a was chosen as the standard substrate for the optimization of asymmetric transfer hydrogenation. Based on previous studies, the ATH was set at $40{ }^{\circ} \mathrm{C}$ in acetonitrile with a (1:1) molar mixture of formic acid and triethylamine as the hydrogen source. Under these conditions, a set of commercially available $\mathrm{Ru}(\mathrm{II})$ catalysts and a $\mathrm{Rh}(\mathrm{III})$ complex were screened (Table 1 ). After $3 \mathrm{~h}$ with a catalyst loading of $0.5 \mathrm{~mol} \%$, the Ru and Rh catalysts allowed a full conversion of the 
$\alpha$-fluoro ketone into the corresponding fluoro alcohol 3a. All the complexes delivered excellent isolated yields and enantiomeric excesses for the desired fluorohydrin $3 a$ (entries 1 to 4$)$. The main difference appeared in the diastereoselectivity outcome. Whereas $(R, R)$ Rh-teth-TsDPEN $((R, R)$-A; entry 1$)$ led to a moderate 79:21 dr, $(R, R)-R u(p$-cymene)TsDPEN $((R, R)-\mathbf{B}$; entry 2$),(R, R)-\mathrm{Ru}$ (mesitylene)TsDPEN $((R, R)$-C; entry 3$)$ and $(R, R)$-TsDENEB $((R, R)$-D; entry 4$)$, all gave an excellent diastereoisomeric ratio dr of $97: 3$ or $98: 2$, indicating that the reaction was neither affected by the nature of the arene ligand nor the tethered or untethered characteristic of the complex. From this series of results, the $(R, R)$-TsDENEB $((R, R)-\mathrm{D})$ catalyst was chosen for the optimization conditions.

Table 1. Catalyst screening for the ATH/DKR ${ }^{\mathrm{a}}$.<smiles>CC(C)(C)OC(=O)N1CC(F)C(=O)c2ccccc21</smiles>

2a

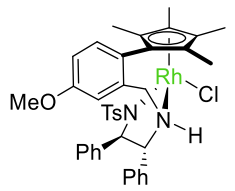

A

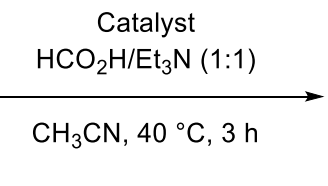

$\mathrm{CH}_{3} \mathrm{CN}, 40^{\circ} \mathrm{C}, 3 \mathrm{~h}$

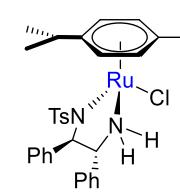

B<smiles>CC(C)(C)OC(=O)N1C[C@H](F)[C@H](O)c2ccccc21</smiles>

3a

\begin{tabular}{ccccc}
\hline Entry & Catalyst & Yield $^{\mathbf{b}}$ & $\mathbf{d r}^{\mathbf{c}}$ & $\mathbf{e e}^{\mathbf{d}}$ \\
\hline 1 & A & $95 \%$ & $79: 21$ & $95 \%$ \\
2 & $\mathrm{~B}$ & $93 \%$ & $97: 3$ & $99 \%$ \\
3 & $\mathrm{C}$ & $93 \%$ & $97: 3$ & $98 \%$ \\
4 & $\mathrm{D}$ & $94 \%$ & $98: 2$ & $>99 \%$
\end{tabular}

a Conditions: 2a $(0.32 \mathrm{mmol})$, catalyst $(0.5 \mathrm{~mol} \%), \mathrm{HCO}_{2} \mathrm{H} / \mathrm{Et}_{3} \mathrm{~N}(1: 1)$ (6 equiv), $\mathrm{CH}_{3} \mathrm{CN}(1 \mathrm{~mL}), 40{ }^{\circ} \mathrm{C}, 3 \mathrm{~h}$ ${ }^{b}$ Isolated yield. ${ }^{c}$ Determined by ${ }^{1} \mathrm{H}-\mathrm{NMR}$ analysis of the crude mixture. ${ }^{\mathrm{d}}$ Determined by SFC analysis. See Supplementary Materials.

The solvent of the asymmetric transfer hydrogenation was then investigated. By using a catalyst loading of $0.5 \mathrm{~mol} \%$ of $(R, R)-\mathrm{D}$, formic acid/triethylamine (1:1) mixture and a reaction time of $3 \mathrm{~h}$, we tested a series of different solvents (Table 2). Several polar aprotic $\left(\mathrm{CH}_{3} \mathrm{CN}, \mathrm{CH}_{2} \mathrm{Cl}_{2}\right.$, THF, Me-THF and EtOAc) and polar protic (MeOH, $\mathrm{PrOH}$ and HFIP) as well as aromatic solvents were screened (toluene, chlorobenzene and trifluoromethylbenzene). The diastereo- and enantioselectivities remained mostly unchanged in all the tests. The conversion rate and the yield showed to be highly dependent on the solvent used. Isopropanol and acetonitrile led to $95 \%$ and $100 \%$ conversions, respectively, with excellent stereoselectivities in both solvents, acetonitrile slightly surpassing isopropanol in all of them. This prompted us to set acetonitrile as the solvent for the asymmetric transfer hydrogenation.

We continued the optimization by varying the hydrogen source of the reaction (Table 3). The acid-to-base ratio was examined (entries 1 to 4 ). Changing the formic acid to triethylamine ratio from 1:1 to 2:5 (entry 2) showed no impact on the reaction. However, switching to a $\mathrm{HCO}_{2} \mathrm{H} / \mathrm{Et}_{3} \mathrm{~N}$ ratio of 5:2 (entry 3) was detrimental to the diastereoselectivity which dropped to a 54:46 dr. Moreover, when this ratio was further increased to 12:1 (entry 4), no conversion was observed. This strong influence of the acid/base ratio of the hydrogen donor reflects the one that was observed in the ATH/DKR of 3-fluorochromanones [6]. Other organic bases associated to formic acid (DBU, entry 5, and DABCO, entry 6) as well as formate salts (ammonium formate and calcium formate, entries 7 and 8, respectively) were screened, all giving lower conversions and similar stereoselectivities than $\mathrm{Et}_{3} \mathrm{~N}$. Finally, the 
catalyst loading (0.2 mol\%; entry 9 ) and the reaction temperature (room temperature; entry $10)$ were lowered. Both tests gave lower conversions in spite of longer reaction times, so we set the optimized conditions for the ATH/DKR as $0.5 \mathrm{~mol} \%$ of $(R, R)-\mathrm{D}$ as the catalyst, $\mathrm{HCO}_{2} \mathrm{H} / \mathrm{Et}_{3} \mathrm{~N}(1: 1)$ mixture as the hydrogen donor, acetonitrile as the solvent and $40{ }^{\circ} \mathrm{C}$ as the reaction temperature.

Table 2. Solvent optimization ${ }^{\text {a }}$.<smiles>CC(C)(C)OC(=O)N1CC(F)C(=O)c2ccccc21</smiles>

$2 \mathbf{a}$

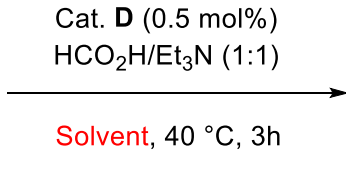

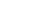<smiles>CC(C)(C)OC(=O)N1CC(F)=C(O)c2ccccc21</smiles>

$3 a$

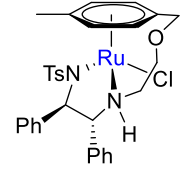

Cat. D

\begin{tabular}{cccccc}
\hline Entry & Solvent & Conversion ${ }^{\mathbf{b}}$ & Yield $^{\mathbf{c}}$ & dr $^{\mathbf{b}}$ & ee $^{\mathbf{d}}$ \\
\hline 1 & $\mathrm{CH}_{3} \mathrm{CN}$ & $100 \%$ & $93 \%$ & $98: 2$ & $>99 \%$ \\
2 & $\mathrm{CH}_{2} \mathrm{Cl}_{2}$ & $83 \%$ & $78 \%$ & $98: 2$ & $95 \%$ \\
3 & $\mathrm{EtOAc}$ & $48 \%$ & $46 \%$ & $98: 2$ & $98 \%$ \\
4 & $\mathrm{THF}$ & $38 \%$ & $36 \%$ & $98: 2$ & $>99 \%$ \\
5 & $\mathrm{Me}-\mathrm{THF}$ & $18 \%$ & $15 \%$ & $96: 4$ & $98 \%$ \\
6 & Toluene & $26 \%$ & $26 \%$ & $97: 3$ & $96 \%$ \\
7 & $\mathrm{Cl-benzene}$ & $43 \%$ & $42 \%$ & $97: 3$ & $94 \%$ \\
8 & $\mathrm{CF}_{3}$-benzene & $60 \%$ & $57 \%$ & $98: 2$ & $96 \%$ \\
9 & $\mathrm{MeOH}$ & $76 \%$ & $73 \%$ & $98: 2$ & $96 \%$ \\
10 & iPrOH & $95 \%$ & $94 \%$ & $98: 2$ & $98 \%$ \\
11 & HFIP & $26 \%$ & $24 \%$ & $98: 2$ & $>99 \%$ \\
\hline
\end{tabular}

a Conditions: 2a (0.32 mmol), catalyst $(0.5 \mathrm{~mol} \%), \mathrm{HCO}_{2} \mathrm{H} / \mathrm{Et}_{3} \mathrm{~N}(1: 1)\left(6\right.$ equiv), solvent $(1 \mathrm{~mL}), 40{ }^{\circ} \mathrm{C}, 3 \mathrm{~h}$.

${ }^{b}$ Determined by ${ }^{1} \mathrm{H}-\mathrm{NMR}$ analysis of the crude mixture. ${ }^{\mathrm{c}}$ Isolated yield. ${ }^{\mathrm{d}}$ Determined by SFC analysis.

Table 3. Survey of the hydrogen donor ${ }^{\mathrm{a}}$.<smiles>O=C1c2ccccc2N(C(=O)OCc2ccccc2)CC1F</smiles>

$2 \mathbf{a}$

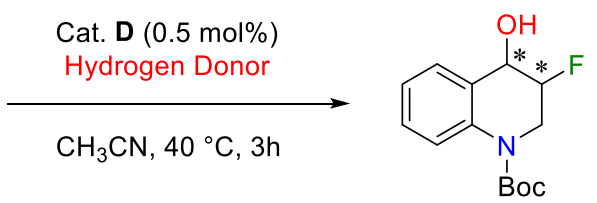

$3 a$

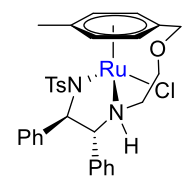

Cat. D

\begin{tabular}{|c|c|c|c|c|c|}
\hline Entry & Hydrogen Donor & Conversion & Yield $^{b}$ & $\mathrm{dr}^{\mathrm{c}}$ & $e e^{d}$ \\
\hline 1 & $\mathrm{HCO}_{2} \mathrm{H} / \mathrm{Et}_{3} \mathrm{~N}(1: 1)$ & $100 \%$ & $93 \%$ & $98: 2$ & $>99 \%$ \\
\hline 2 & $\mathrm{HCO}_{2} \mathrm{H} / \mathrm{Et}_{3} \mathrm{~N}(2: 5)$ & $100 \%$ & $95 \%$ & $98: 2$ & $99 \%$ \\
\hline 3 & $\mathrm{HCO}_{2} \mathrm{H} / \mathrm{Et}_{3} \mathrm{~N}(5: 2)$ & $100 \%$ & $94 \%$ & $54: 46$ & $92 \%$ \\
\hline 4 & $\mathrm{HCO}_{2} \mathrm{H} / \mathrm{Et}_{3} \mathrm{~N}(12: 1)$ & $0 \%$ & - & - & - \\
\hline 5 & $\mathrm{HCO}_{2} \mathrm{H} / \mathrm{DBU}(1: 1)$ & $90 \%$ & $88 \%$ & $98: 2$ & $99 \%$ \\
\hline 6 & $\mathrm{HCO}_{2} \mathrm{H} / \mathrm{DABCO}(1: 1)$ & $75 \%$ & $73 \%$ & $98: 2$ & $98 \%$ \\
\hline 7 & $\mathrm{HCO}_{2} \mathrm{NH}_{4}$ & $35 \%$ & $34 \%$ & $98: 2$ & $99 \%$ \\
\hline $8^{e}$ & $\left(\mathrm{HCO}_{2}\right)_{2} \mathrm{Ca}$ & $20 \%$ & $16 \%$ & $98: 2$ & $98 \%$ \\
\hline $9^{f}$ & $\mathrm{HCO}_{2} \mathrm{H} / \mathrm{Et}_{3} \mathrm{~N}(1: 1)$ & $54 \%$ & $53 \%$ & $98: 2$ & $>99 \%$ \\
\hline $10^{g}$ & $\mathrm{HCO}_{2} \mathrm{H} / \mathrm{Et}_{3} \mathrm{~N}(1: 1)$ & $30 \%$ & $23 \%$ & $98: 2$ & $>99 \%$ \\
\hline
\end{tabular}

a Conditions: 2a $(0.32 \mathrm{mmol})$, catalyst $(0.5 \mathrm{~mol} \%), \mathrm{HCO}_{2} \mathrm{H} / \mathrm{Et}_{3} \mathrm{~N}$ (1:1) (6 equiv), $\mathrm{CH}_{3} \mathrm{CN}(1 \mathrm{~mL}), 40{ }^{\circ} \mathrm{C}, 3 \mathrm{~h}$

b Isolated yield. ${ }^{c}$ Determined by ${ }^{1} \mathrm{H}-\mathrm{NMR}$ analysis of the crude mixture. ${ }^{\mathrm{d}}$ Determined by SFC analysis. e $\mathrm{CH}_{3} \mathrm{CN} / \mathrm{H}_{2} \mathrm{O}(10: 1)$ was used as solvent. ${ }^{\mathrm{f}}$ Catalyst loading of $0.2 \mathrm{~mol} \%$ and reaction time of $24 \mathrm{~h} .{ }^{\mathrm{g}}$ Reaction run at r. t. $\left(22^{\circ} \mathrm{C}\right)$ for $24 \mathrm{~h}$

Once the aforementioned conditions were identified, we subjected the family of substituted tert-butyl 3-fluoro tetrahydroquinoline-4-ones carboxylates $(\mathbf{2} \mathbf{a}-\mathbf{j})$ previously synthesized to the asymmetric transfer hydrogenation. The scope (Scheme 3 ) showed that the fluoro dihydrotetrahydroquinolin-4-ones bearing electron-donating or electron-withdrawing 
groups all led to good yields (90-96\%), diastereo- (94:6 to 99:1 dr) and enantioselectivities (99-> 99\% ee) to the corresponding fluorohydrins (3a-j). Notably, the asymmetric transfer hydrogenation tolerated substituents such as chlorine, bromine or iodine along with other groups such as methyl, methoxy and trifluoromethyl. Concerning the outcome of the reaction, the two substrates bearing a methoxy-group in position 7 of the aromatic ring ( $\mathbf{2 d}$ and $\mathbf{2 e}$ ) gave a slightly lower diastereoselectivity compared to the rest of the dihydroquinolin-4-ones. The ATH of compound $\mathbf{2 e}$, possessing two methoxy groups in positions 6 and 7, also required a higher catalyst loading $(1.0 \mathrm{~mol} \%)$ and a longer reaction time $(24 \mathrm{~h})$ to achieve a full conversion.

The absolute configuration of compound 3a was unambiguously assigned as ( $3 R$, $4 S$ ) by the X-ray crystallographic analysis. We conjectured that the other products of the developed ATH/DKR reaction led to 3-fluoro-dihydrotetrahydroquinolin-4-ols derivatives with the same absolute configuration (Scheme 3).<smiles>CC(C)(C)OC(=O)N1CC(F)C(=O)c2cc[R][c]c21</smiles>

2

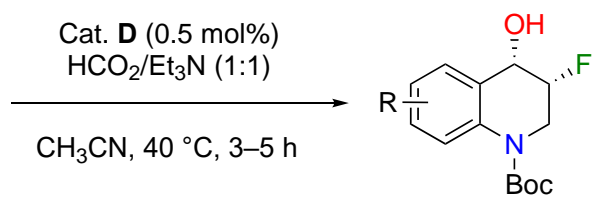

3

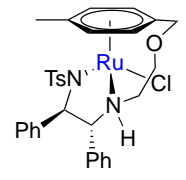

Cat. D

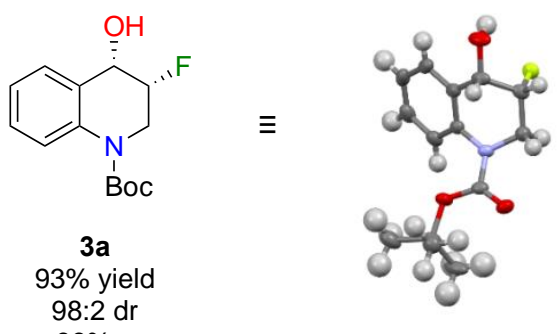<smiles>Cc1ccc2c(c1)[C@H](O)[C@H](F)CN2C(=O)OC(C)(C)C</smiles>

3b

91\% yield

98:2 dr $>99 \%$ ee<smiles>COc1ccc2c(c1)[C@@H](O)[C@H](F)CN2C(=O)OC(C)(C)C</smiles>

$3 c^{a}$

95\% yield

98:2 dr $>99 \%$ ee<smiles>COc1ccc2c(c1)N(C(=O)c1ccccc1)C[C@H](F)[C@H]2O</smiles>

$3 d^{a}$

95\% yield

95:5 dr

$>99 \%$ ee<smiles>COc1cc2c(cc1OC)N(C(=O)OC(C)(C)C)C[C@@H](F)[C@H]2O</smiles>

$3 \mathbf{e}^{\mathrm{b}}$

$90 \%$ yield

94:6 dr $99 \%$ ee<smiles>CC(C)(C)OC(=O)N1C[C@H](F)[C@H](O)c2cc(F)ccc21</smiles>

$3 f^{\mathrm{c}}$

$70 \%$ yield $(74 \%$ conversion)

$98: 2 \mathrm{dr}$

$>99 \%$ ee<smiles>CC(C)(C)OC(=O)OC(=O)c1ccc(C(F)(F)F)cc1[C@@H](O)[C@H](F)C(=O)O</smiles>

$3 \mathbf{g}$

97\% yield

98:2 dr $>99 \%$ ee<smiles>CC(C)(C)OC(=O)OC(C)(C)C</smiles>

3h 96\% yield 99:1 dr $>99 \%$ ee<smiles>CC(C)(C)OC(=O)N1C[C@H](F)[C@H](O)c2cc(Br)ccc21</smiles>

$3 \mathbf{i}$

95\% yield

98:2 dr $>99 \%$ ee<smiles>CC(C)(C)OC(=O)N1C[C@H](F)[C@H](O)c2cc(I)ccc21</smiles>

3j

$94 \%$ yield

98:2 dr

$>99 \%$ ee

Scheme 3. Scope of the reaction. Unless specified, the reaction time is $3 \mathrm{~h}$. ${ }^{\text {a }}$ Reaction time of $5 \mathrm{~h}$.

b $1.0 \mathrm{~mol} \%$ of catalyst and reaction time of $24 \mathrm{~h} .{ }^{\mathrm{c}}$ Reaction time of $24 \mathrm{~h}$.

The usefulness of the studied asymmetric transfer hydrogenation was evaluated by performing the reaction on a gram-scale with substrate 2a (Scheme 4). $1.0 \mathrm{~g}$ of tert-butyl 3-fluoro-dihydrotetrahydroquinolin-4-one carboxylate was subjected to the optimized 
reaction conditions which led to the cis-fluorohydrin 3a in 96\% yield, 97:3 dr and > 99\% ee. In addition, the post-functionalization of compound $3 \mathbf{j}$ using a Sonogashira cross-coupling reaction with phenylacetylene was carried out in the presence of $\mathrm{PdCl}_{2}\left(\mathrm{PPh}_{3}\right)_{2}$ and $\mathrm{CuI}$ to afford the corresponding alkyne 4 in $68 \%$ yield. Finally, $N$-Boc deprotection of compound 3a was readily achieved by heating in dioxane $/ \mathrm{H}_{2} \mathrm{O}$ and the desired amine 5 was isolated in $90 \%$ yield.<smiles>CC(C)(C)OC(=O)N1CC(F)C(=O)c2ccccc21</smiles>

2a $1.0 \mathrm{~g}$<smiles>CC(C)(C)OC(=O)N1C[C@H](F)[C@H](O)c2cc(I)ccc21</smiles><smiles>CC(C)(C)OC(=O)N1C[C@H](F)[C@H](O)c2ccccc21</smiles>

3a

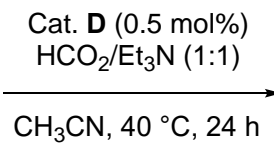

$\mathrm{CH}_{3} \mathrm{CN}, 40^{\circ} \mathrm{C}, 24 \mathrm{~h}$

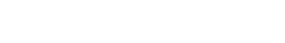<smiles>CC(C)(C)OC(=O)N1C[C@H](F)[C@H](O)c2ccccc21</smiles>

$3 a$ $96 \%$ yield 97:3 dr $>99 \%$ ee

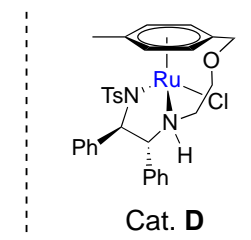

Cat. D

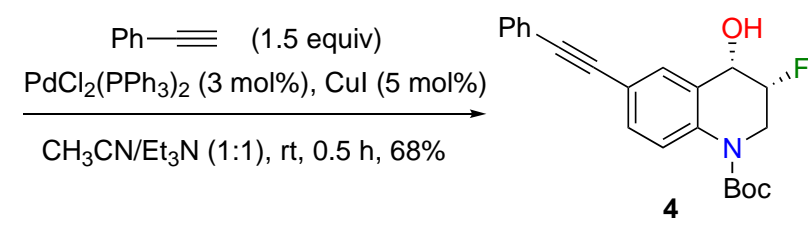

$\underset{\text { reflux, } 34 \mathrm{~h}, 90 \%}{\stackrel{\text { dioxane } / \mathrm{H}_{2} \mathrm{O}(3: 5)}{\longrightarrow}}$<smiles>O[C@H]1C[C@H](F)c2ccccc2N1</smiles>

Scheme 4. Scale-up, functionalization and deprotection experiments.

\section{Materials and Methods}

\subsection{General Information}

All air- and/or water-sensitive reactions were carried out under an argon atmosphere. $\mathrm{THF}, \mathrm{CH}_{2} \mathrm{Cl}_{2}$ and toluene were dried over alumina columns in a solvent purification apparatus (Innovative Technology, Oldham, UK). Methanol, isopropanol, chlorobenzene, trifluoromethylbenzene, ethyl acetate and acetonitrile from Sigma-Aldrich (Darmstadt, Germany) were used without further purification. Hexafluoroisopropanol (HFIP) was purchased from TCI and was used without further purification. Formic acid/triethylamine (1:1) mixture was purchased from Fluka or Alfa Aesar and was used without further purification. Reactions were monitored by thin layer chromatography carried out on precoated silica gel plates (Merck 60F254, Darmstadt, Germany) and revealed with either an ultraviolet lamp $(\lambda=254 \mathrm{~nm})$ or a potassium permanganate solution. Proton nuclear magnetic resonance $\left({ }^{1} \mathrm{H}-\mathrm{NMR}\right)$ spectra were recorded using a Bruker AC $400(400 \mathrm{MHz})$. The chemical shifts are expressed in parts per million (ppm) referenced to residual chloroform (7.26 ppm). Data are reported as follows: chemical shifts $(\delta)$, multiplicity (recorded as s, singlet; d, doublet; $\mathrm{t}$, triplet; q, quadruplet; quint, quintuplet; sext, sextuplet; hept, heptuplet; $\mathrm{m}$, multiplet and br, broad), coupling constants and integration. Carbon-13 nuclear magnetic resonance $\left({ }^{13} \mathrm{C}-\mathrm{NMR}\right)$ spectra were recorded using a Bruker AC $400(101 \mathrm{MHz})$. The chemical shifts are expressed in parts per million (ppm) relative to the centre line of the triplet at $77.16 \mathrm{ppm}$ for $\mathrm{CDCl}_{3}$. Melting points (m. p.) were determined on a Köfler melting point apparatus. Optical rotations were measured on a Jasco P-1010 polarimeter. High resolution mass spectrometric (HRMS) analyses were measured on LTQ-Orbitrap (Thermo Fisher Scientific, Waltham, Massachusetts, US) at Sorbonne Université. 


\subsection{Method for the Synthesis of Compounds $\mathbf{2}$}

To a $0{ }^{\circ} \mathrm{C}$ solution of LiHMDS (1.0 M in THF, 1.05 equiv) in a round-bottom tube set under argon, was slowly added a solution of the ketone (1.2 mmol, 1.0 equiv) in THF ( $2 \mathrm{~mL})$ over $5 \mathrm{~min}$. The mixture was stirred at $0{ }^{\circ} \mathrm{C}$ for $1.5 \mathrm{~h}$. The resulting solution was then added dropwise over $5 \mathrm{~min}$ via a cannula to a $-78^{\circ} \mathrm{C}$ solution of NFSI (1.4 mmol, 1.2 equiv) in THF ( $4 \mathrm{~mL})$. The reaction mixture was stirred at $-78{ }^{\circ} \mathrm{C}$ for $30 \mathrm{~min}$ and then allowed to come to room temperature overnight. The reaction was diluted with $3 \mathrm{~mL}$ of $\mathrm{CH}_{2} \mathrm{Cl}_{2}$, quenched with $5 \mathrm{~mL}$ of saturated $\mathrm{NH}_{4} \mathrm{Cl}$ aqueous solution and extracted with $\mathrm{CH}_{2} \mathrm{Cl}_{2}(3 \times$ $10 \mathrm{~mL}$ ). The combined organic layers were washed with brine, dried over $\mathrm{MgSO}_{4}$, filtered, and concentrated under reduced pressure [25]. The pure products were isolated by flash column chromatography on silica gel (see Supplementary Materials for NMR spectra).

tert-Butyl 3-fluoro-4-oxo-3,4-dihydroquinoline-1(2H)-carboxylate (2a). $2.0 \mathrm{~g}$ of tert-butyl 4-oxo-3,4-dihydroquinoline-1(2H)-carboxylate (8.09 mmol, 1.0 equiv) were used following the described procedure. Purification via flash column chromatography on silica gel (95:5 petroleum ether/ethyl acetate) yielded $1.56 \mathrm{~g}$ of $2 \mathrm{a}$ as a white solid (73\%). m.p. $96-100{ }^{\circ} \mathrm{C}$. ${ }^{1} \mathrm{H}-\mathrm{NMR}(400 \mathrm{MHz}$, Chloroform- $d$ ) $\delta 8.03(\mathrm{dd}, J=8.0,1.6 \mathrm{~Hz}, 1 \mathrm{H}), 7.81(\mathrm{dd}, J=8.5,1.0 \mathrm{~Hz}$, $1 \mathrm{H}), 7.54(\mathrm{ddd}, J=8.7,7.2,1.7 \mathrm{~Hz}, 1 \mathrm{H}), 7.20(\mathrm{td}, J=7.6,1.0 \mathrm{~Hz}, 1 \mathrm{H}), 5.10(\mathrm{ddd}, J=46.9,10.5$, $4.7 \mathrm{~Hz}, 1 \mathrm{H}), 4.63(\mathrm{td}, J=13.0,4.7 \mathrm{~Hz}, 1 \mathrm{H}), 4.04(\mathrm{ddd}, J=13.3,10.6,4.8 \mathrm{~Hz}, 1 \mathrm{H}), 1.57(\mathrm{~s}, 9 \mathrm{H})$. ${ }^{19}$ F-NMR (376 MHz, Chloroform- $d$ ) $\delta-198.77 .{ }^{13} \mathrm{C}-\mathrm{NMR}(101 \mathrm{MHz}$, Chloroform- $d$ ) $\delta 189.7$ $\left(\mathrm{C}-\mathrm{F},{ }^{2} J_{\mathrm{CF}}=14.1 \mathrm{~Hz}\right), 189.6\left(\mathrm{C}-\mathrm{F},{ }^{2} J_{\mathrm{CF}}=14.1 \mathrm{~Hz}\right), 152.8,144.1,135.0,128.1,124.6,123.8,123.2$, $88.1\left(\mathrm{C}-\mathrm{F},{ }^{1} J_{\mathrm{CF}}=190.9 \mathrm{~Hz}\right), 86.2\left(\mathrm{C}-\mathrm{F},{ }^{1} J_{\mathrm{CF}}=190.9 \mathrm{~Hz}\right), 83.2,48.8\left(\mathrm{C}-\mathrm{F},{ }^{2} J_{\mathrm{CF}}=27.3 \mathrm{~Hz}\right), 48.5$ $\left(\mathrm{C}-\mathrm{F},{ }^{2} J_{\mathrm{CF}}=27.3 \mathrm{~Hz}\right), 28.3$. HRMS (APCI): $m / z[\mathrm{M}+\mathrm{H}]^{+}$calcd. for $\mathrm{C}_{14} \mathrm{H}_{17} \mathrm{FNO}_{3} 266.1187$, found 266.1186 .

tert-Butyl 3-fluoro-6-methyl-4-oxo-3,4-dihydroquinoline-1(2H)-carboxylate (2b). $550 \mathrm{mg}$ of tert-butyl 6-methyl-4-oxo-3,4-dihydroquinoline-1(2H)-carboxylate (2.10 mmol, 1.0 equiv) were used following the described procedure. Purification via flash column chromatography on silica gel (95:5 petroleum ether/ethyl acetate) yielded $470 \mathrm{mg}$ of $\mathbf{2 b}$ as a white solid (80\%). m.p. $122-126{ }^{\circ} \mathrm{C} .{ }^{1} \mathrm{H}-\mathrm{NMR}(400 \mathrm{MHz}$, Chloroform- $d) \delta 7.75(\mathrm{~s}, 1 \mathrm{H}), 7.62(\mathrm{~d}$, $J=8.5 \mathrm{~Hz}, 1 \mathrm{H}), 7.28(\mathrm{dd}, J=9.8,3.4 \mathrm{~Hz}, 1 \mathrm{H}), 5.01(\mathrm{ddd}, J=46.9,10.6,4.7 \mathrm{~Hz}, 1 \mathrm{H}), 4.55$ $(\mathrm{tdd}, J=13.2,4.7,0.9 \mathrm{~Hz}, 1 \mathrm{H}), 3.93(\mathrm{ddd}, J=13.5,10.6,4.8 \mathrm{~Hz}, 1 \mathrm{H}), 2.28(\mathrm{~s}, 3 \mathrm{H}), 1.49(\mathrm{~s}, 9 \mathrm{H})$. ${ }^{19}$ F-NMR (376 MHz, Chloroform-d) $\delta-198.75 .{ }^{13} \mathrm{C}-\mathrm{NMR}(101 \mathrm{MHz}$, Chloroform-d) $\delta 189.9$ $\left(\mathrm{C}-\mathrm{F},{ }^{2} J_{\mathrm{CF}}=14.1 \mathrm{~Hz}\right), 189.8\left(\mathrm{C}-\mathrm{F},{ }^{2} J_{\mathrm{CF}}=14.1 \mathrm{~Hz}\right), 152.9,141.8,136.0,134.5,127.8,123.7,123.0$, $88.2\left(\mathrm{C}-\mathrm{F},{ }^{1} J_{\mathrm{CF}}=190.9 \mathrm{~Hz}\right), 86.3\left(\mathrm{C}-\mathrm{F},{ }^{1} J_{\mathrm{CF}}=190.9 \mathrm{~Hz}\right), 83.0,48.8\left(\mathrm{C}-\mathrm{F},{ }^{2} J_{\mathrm{CF}}=27.3 \mathrm{~Hz}\right), 48.5$ $\left(\mathrm{C}-\mathrm{F},{ }^{2} J_{\mathrm{CF}}=27.3 \mathrm{~Hz}\right), 28.4,20.7$. HRMS (APCI): $m / z[\mathrm{M}-\mathrm{Boc}+\mathrm{H}]^{+}$calcd. for $\mathrm{C}_{10} \mathrm{H}_{11} \mathrm{FNO}$ 180.0825 , found 180.0819 .

tert-Butyl 3-fluoro-6-methoxy-4-oxo-3,4-dihydroquinoline-1(2H)-carboxylate (2c). $700 \mathrm{mg}$ of tert-butyl 6-methoxy-4-oxo-3,4-dihydroquinoline-1(2H)-carboxylate (2.52 mmol, 1.0 equiv) were used following the described procedure. Purification via flash column chromatography on silica gel (95:5 to 85:15 petroleum ether/ethyl acetate) yielded $487 \mathrm{mg}$ of $2 \mathrm{c}$ as a pale-yellow solid (70\%). m.p. $136-140{ }^{\circ} \mathrm{C} .{ }^{1} \mathrm{H}-\mathrm{NMR}(400 \mathrm{MHz}$, Chloroform- $d) \delta 7.69$ (dd, $J=9.7,2.9 \mathrm{~Hz}, 1 \mathrm{H}), 7.43(\mathrm{dd}, J=7.0,4.2 \mathrm{~Hz}, 1 \mathrm{H}), 7.11(\mathrm{dt}, J=12.5,8.2 \mathrm{~Hz}, 1 \mathrm{H}), 5.08(\mathrm{ddd}$, $J=46.8,17.6,13.6 \mathrm{~Hz}, 1 \mathrm{H}), 4.60(\mathrm{td}, J=13.2,8.9 \mathrm{~Hz}, 1 \mathrm{H}), 4.01(\mathrm{ddt}, J=15.3,10.6,3.2 \mathrm{~Hz}$, 1H), 3.82 (s, 3H), 1.54 (s, 9H). ${ }^{19}$ F-NMR (376 MHz, Chloroform-d) $\delta-198.57 .{ }^{13} \mathrm{C}-\mathrm{NMR}$ $\left(101 \mathrm{MHz}\right.$, Chloroform-d) $\delta 189.7\left(\mathrm{C}-\mathrm{F},{ }^{2} J_{\mathrm{CF}}=15.2 \mathrm{~Hz}\right), 189.5\left(\mathrm{C}-\mathrm{F},{ }^{2} J_{\mathrm{CF}}=15.2 \mathrm{~Hz}\right), 156.5$, $152.9,137.9,125.5,124.0,123.5,108.9,88.7\left(\mathrm{C}-\mathrm{F},{ }^{1} J_{\mathrm{CF}}=190.9 \mathrm{~Hz}\right), 86.4\left(\mathrm{C}-\mathrm{F},{ }^{1} J_{\mathrm{CF}}=190.9 \mathrm{~Hz}\right)$, $82.9,55.8,48.9\left(\mathrm{C}-\mathrm{F},{ }^{2} J_{\mathrm{CF}}=27.3 \mathrm{~Hz}\right), 48.7\left(\mathrm{C}-\mathrm{F},{ }^{2} J_{\mathrm{CF}}=27.3 \mathrm{~Hz}\right), 28.3$. HRMS (APCI): $m / z$ [M - Boc $+\mathrm{H}]^{+}$calcd. for $\mathrm{C}_{10} \mathrm{H}_{11} \mathrm{FNO}_{2}$ 196.0774, found 196.0769.

tert-Butyl 3-fluoro-7-methoxy-4-oxo-3,4-dihydroquinoline-1(2H)-carboxylate (2d). $313 \mathrm{mg}$ of tert-butyl 7-methoxy-4-oxo-3,4-dihydroquinoline-1(2H)-carboxylate (1.13 mmol, 1.0 equiv) were used following the described procedure. Purification via flash column chromatography on silica gel (95:5 to 85:15 petroleum ether/ethyl acetate) yielded $247 \mathrm{mg}$ of $\mathbf{2 d}$ as a pale-yellow solid (74\%). m.p. $93-97{ }^{\circ} \mathrm{C} .{ }^{1} \mathrm{H}-\mathrm{NMR}(400 \mathrm{MHz}$, Chloroform- $d) \delta 7.98$ (dd, 
$J=9.0,1.3 \mathrm{~Hz}, 1 \mathrm{H}), 7.37(\mathrm{~d}, J=2.4 \mathrm{~Hz}, 1 \mathrm{H}), 6.74(\mathrm{dd}, J=8.9,2.4 \mathrm{~Hz}, 1 \mathrm{H}), 5.04(\mathrm{ddd}, J=47.0$, $10.3,4.6 \mathrm{~Hz}, 1 \mathrm{H}), 4.58(\mathrm{td}, J=13.4,4.6 \mathrm{~Hz}, 1 \mathrm{H}), 4.03(\mathrm{ddd}, J=13.2,10.3,5.2 \mathrm{~Hz}, 1 \mathrm{H}), 3.88$ $(\mathrm{s}, 3 \mathrm{H}), 1.58(\mathrm{~s}, 9 \mathrm{H}) .{ }^{19} \mathrm{~F}-\mathrm{NMR}\left(376 \mathrm{MHz}\right.$, Chloroform- $d$ ) $\delta-199.06 .{ }^{13} \mathrm{C}-\mathrm{NMR}(101 \mathrm{MHz}$, Chloroform-d) $\delta 188.3\left(\mathrm{C}-\mathrm{F},{ }^{2} J_{\mathrm{CF}}=15.2 \mathrm{~Hz}\right), 188.1\left(\mathrm{C}-\mathrm{F},{ }^{2} J_{\mathrm{CF}}=15.2 \mathrm{~Hz}\right), 165.1,152.7,146.0$, $130.2,116.9,112.0,107.7,87.8\left(\mathrm{C}-\mathrm{F},{ }^{1} J_{\mathrm{CF}}=189.9 \mathrm{~Hz}\right), 85.9\left(\mathrm{C}-\mathrm{F},{ }^{1} J_{\mathrm{CF}}=189.9 \mathrm{~Hz}\right), 83.2,55.9$, $48.9\left(\mathrm{C}-\mathrm{F},{ }^{2} J_{\mathrm{CF}}=27.3 \mathrm{~Hz}\right), 48.7\left(\mathrm{C}-\mathrm{F},{ }^{2} J_{\mathrm{CF}}=27.3 \mathrm{~Hz}\right), 28.4$. HRMS (APCI): $m / z[\mathrm{M}+\mathrm{H}]^{+}$ calcd. for $\mathrm{C}_{15} \mathrm{H}_{19} \mathrm{FNO}_{4} 296.1293$, found 296.1295.

tert-Butyl 3-fluoro-6,7-dimethoxy-4-oxo-3,4-dihydroquinoline-1(2H)-carboxylate (2e). $330 \mathrm{mg}$ of tert-butyl 6,7-dimethoxy-4-oxo-3,4-dihydroquinoline-1(2H)-carboxylate $(1.07 \mathrm{mmol}$, 1.0 equiv) were used following the described procedure. Purification via flash column chromatography on silica gel (90:10 to 80:20 petroleum ether/ethyl acetate) yielded $257 \mathrm{mg}$ of 2e as a pale-yellow solid (74\%). m.p. $168-172{ }^{\circ} \mathrm{C} .{ }^{1} \mathrm{H}-\mathrm{NMR}(400 \mathrm{MHz}$, Chloroform- $d$ ) $\delta 7.46-7.36(\mathrm{~m}, 2 \mathrm{H}), 5.05(\mathrm{ddd}, J=47.0,10.1,4.5 \mathrm{~Hz}, 1 \mathrm{H}), 4.56(\mathrm{ddd}, J=14.3,13.2,4.5 \mathrm{~Hz}$, $1 \mathrm{H}), 4.06(\mathrm{ddd}, J=13.3,10.1,5.4 \mathrm{~Hz}, 1 \mathrm{H}), 3.96(\mathrm{~s}, 3 \mathrm{H}), 3.91(\mathrm{~s}, 3 \mathrm{H}), 1.57(\mathrm{~s}, 9 \mathrm{H}) .{ }^{19} \mathrm{~F}-\mathrm{NMR}$ (376 MHz, Chloroform- $d$ ) $\delta-198.64 .{ }^{13} \mathrm{C}-\mathrm{NMR}(101 \mathrm{MHz}$, Chloroform- $d$ ) $\delta 188.3$ (C-F, $\left.{ }^{2} J_{\mathrm{CF}}=15.2 \mathrm{~Hz}\right), 188.1\left(\mathrm{C}-\mathrm{F},{ }^{2} J_{\mathrm{CF}}=15.2 \mathrm{~Hz}\right), 154.8,152.8,146.6,140.0,116.2,108.0,106.3,87.9$ $\left(\mathrm{C}-\mathrm{F},{ }^{1} J_{\mathrm{CF}}=188.9 \mathrm{~Hz}\right), 86.0\left(\mathrm{C}-\mathrm{F},{ }^{1} J_{\mathrm{CF}}=188.9 \mathrm{~Hz}\right), 83.0,56.4,56.3,49.4\left(\mathrm{C}-\mathrm{F},{ }^{2} J_{\mathrm{CF}}=27.3 \mathrm{~Hz}\right)$, $49.1\left(\mathrm{C}-\mathrm{F},{ }^{2} J_{\mathrm{CF}}=27.3 \mathrm{~Hz}\right)$, 28.4. HRMS (APCI): $m / z[\mathrm{M}+\mathrm{H}]^{+}$calcd. for $\mathrm{C}_{16} \mathrm{H}_{21} \mathrm{FNO}_{5}$ 326.1398, found 326.1399.

tert-Butyl 3,6-difluoro-4-oxo-3,4-dihydroquinoline-1(2H)-carboxylate (2f). $500 \mathrm{mg}$ of tertbutyl 6-fluoro-4-oxo-3,4-dihydroquinoline-1 $(2 \mathrm{H})$-carboxylate (1.88 $\mathrm{mmol}, 1.0$ equiv) were used following the described procedure. Purification via flash column chromatography on silica gel (95:5 to 85:15 petroleum ether/ethyl acetate) yielded $474 \mathrm{mg}$ of $\mathbf{2} \mathbf{f}$ as a paleyellow solid (86\%). m.p. 89-93 ${ }^{\circ} \mathrm{C} .{ }^{1} \mathrm{H}-\mathrm{NMR}(400 \mathrm{MHz}$, Chloroform- $d$ ) 87.81 (ddd, $J=9.7$, $4.5,2.3 \mathrm{~Hz}, 1 \mathrm{H}), 7.65(\mathrm{dt}, J=8.3,4.3 \mathrm{~Hz}, 1 \mathrm{H}), 7.31-7.20(\mathrm{~m}, 1 \mathrm{H}), 5.10(\mathrm{ddd}, J=46.7,10.0$, $2.7 \mathrm{~Hz}, 1 \mathrm{H}), 4.59(\mathrm{td}, J=13.4,3.8 \mathrm{~Hz}, 1 \mathrm{H}), 4.06(\mathrm{ddd}, J=12.1,5.1,2.5 \mathrm{~Hz}, 1 \mathrm{H}), 1.55(\mathrm{~s}$, 9H). m.p. $89-93{ }^{\circ} \mathrm{C} .{ }^{19} \mathrm{~F}-\mathrm{NMR}\left(376 \mathrm{MHz}\right.$, Chloroform- $d$ ) $\delta-116.65,-198.88 .{ }^{13} \mathrm{C}-\mathrm{NMR}$ $(101 \mathrm{MHz}$, Chloroform- $d) \delta 188.8\left(\mathrm{C}-\mathrm{F},{ }^{2} J_{\mathrm{CF}}=15.2 \mathrm{~Hz}\right), 188.6\left(\mathrm{C}-\mathrm{F},{ }^{2} J_{\mathrm{CF}}=15.2 \mathrm{~Hz}\right), 160.5$ $\left(\mathrm{C}-\mathrm{F},{ }^{1} J_{\mathrm{CF}}=248.5 \mathrm{~Hz}\right), 158.1\left(\mathrm{C}-\mathrm{F},{ }^{1} J_{\mathrm{CF}}=248.5 \mathrm{~Hz}\right), 152.7,140.4,126.1\left(\mathrm{C}-\mathrm{F},{ }^{3} J_{\mathrm{CF}}=7.1 \mathrm{~Hz}\right)$, $126.1\left(\mathrm{C}-\mathrm{F},{ }^{3} J_{\mathrm{CF}}=7.1 \mathrm{~Hz}\right), 124.6\left(\mathrm{C}-\mathrm{F},{ }^{3} J_{\mathrm{CF}}=6.1 \mathrm{~Hz}\right), 124.6\left(\mathrm{C}-\mathrm{F},{ }^{3} J_{\mathrm{CF}}=6.1 \mathrm{~Hz}\right), 122.5$ $\left(\mathrm{C}-\mathrm{F},{ }^{2} J_{\mathrm{CF}}=23.2 \mathrm{~Hz}\right), 122.3\left(\mathrm{C}-\mathrm{F},{ }^{2} J_{\mathrm{CF}}=23.2 \mathrm{~Hz}\right), 113.5\left(\mathrm{C}-\mathrm{F},{ }^{2} J_{\mathrm{CF}}=23.2 \mathrm{~Hz}\right), 113.3(\mathrm{C}-\mathrm{F}$, $\left.{ }^{2} J_{\mathrm{CF}}=23.2 \mathrm{~Hz}\right), 88.0\left(\mathrm{C}-\mathrm{F},{ }^{1} J_{\mathrm{CF}}=191.9 \mathrm{~Hz}\right), 86.1\left(\mathrm{C}-\mathrm{F},{ }^{1} J_{\mathrm{CF}}=191.9 \mathrm{~Hz}\right), 83.4,48.8(\mathrm{C}-\mathrm{F}$, $\left.{ }^{2} J_{\mathrm{CF}}=26.3 \mathrm{~Hz}\right), 48.5\left(\mathrm{C}-\mathrm{F},{ }^{2} J_{\mathrm{CF}}=26.3 \mathrm{~Hz}\right), 28.3$. HRMS (APCI): $m / z[\mathrm{M}-\mathrm{Boc}+\mathrm{H}]^{+}$calcd. for $\mathrm{C}_{9} \mathrm{H}_{8} \mathrm{~F}_{2} \mathrm{NO} 184.0574$, found 184.0569.

tert-Butyl 3-fluoro-4-oxo-6-(trifluoromethyl)-3,4-dihydroquinoline-1(2H)-carboxylate (2g). $700 \mathrm{mg}$ of tert-butyl 4-oxo-6-(trifluoromethyl)-3,4-dihydroquinoline-1(2H)-carboxylate ( $2.22 \mathrm{mmol}, 1.0$ equiv) were used following the described procedure. Purification via flash column chromatography on silica gel ( $95: 5$ to $85: 15$ petroleum ether/ethyl acetate) yielded $335 \mathrm{mg}$ of $2 \mathrm{~g}$ as a white solid (45\%). m.p. $101-105{ }^{\circ} \mathrm{C} .{ }^{1} \mathrm{H}-\mathrm{NMR}(400 \mathrm{MHz}$, Chloroform-d) $\delta 8.29(\mathrm{~d}, J=2.3 \mathrm{~Hz}, 1 \mathrm{H}), 8.03(\mathrm{dd}, J=8.9,0.6 \mathrm{~Hz}, 1 \mathrm{H}), 7.75(\mathrm{dd}, J=7.1$, $2.8 \mathrm{~Hz}, 1 \mathrm{H}), 5.13(\mathrm{ddd}, J=46.7,10.3,4.6 \mathrm{~Hz}, 1 \mathrm{H}), 4.62(\mathrm{ddd}, J=14.3,13.6,4.6 \mathrm{~Hz}, 1 \mathrm{H})$, $4.12(\mathrm{ddd}, J=13.5,10.3,5.1 \mathrm{~Hz}, 1 \mathrm{H}), 1.58(\mathrm{~s}, 9 \mathrm{H}) .{ }^{19} \mathrm{~F}-\mathrm{NMR}(376 \mathrm{MHz}$, Chloroform- $d$ ) $\delta$ -62.76, -199.13. ${ }^{13} \mathrm{C}-\mathrm{NMR}\left(101 \mathrm{MHz}\right.$, Chloroform- $d$ ) $\delta 188.4\left(\mathrm{C}-\mathrm{F},{ }^{2} J_{\mathrm{CF}}=15.2 \mathrm{~Hz}\right), 188.2$ $\left(\mathrm{C}-\mathrm{F},{ }^{2} J_{\mathrm{CF}}=15.2 \mathrm{~Hz}\right), 152.3,146.6,131.3\left(\mathrm{C}-\mathrm{F}^{3}{ }^{3} J_{\mathrm{CF}}=4.0 \mathrm{~Hz}\right), 131.2\left(\mathrm{C}-\mathrm{F},{ }^{3} J_{\mathrm{CF}}=4.0 \mathrm{~Hz}\right), 127.6$ $\left(\mathrm{C}-\mathrm{F},{ }^{1} J_{\mathrm{CF} 3}=272.7 \mathrm{~Hz}\right), 127.2\left(\mathrm{C}-\mathrm{F},{ }^{2} J_{\mathrm{CF} 3}=33.3 \mathrm{~Hz}\right), 126.9\left(\mathrm{C}-\mathrm{F},{ }^{2} J_{\mathrm{CF} 3}=33.3 \mathrm{~Hz}\right), 126.6(\mathrm{C}-\mathrm{F}$, $\left.{ }^{2} J_{\mathrm{CF} 3}=33.3 \mathrm{~Hz}\right), 126.2\left(\mathrm{C}-\mathrm{F}^{2}{ }^{2} J_{\mathrm{CF} 3}=33.3 \mathrm{~Hz}\right), 125.6,124.9\left(\mathrm{C}-\mathrm{F},{ }^{1} J_{\mathrm{CF} 3}=272.7 \mathrm{~Hz}\right), 124.2,122.7$, $122.2\left(\mathrm{C}-\mathrm{F},{ }^{1} J_{\mathrm{CF} 3}=272.7 \mathrm{~Hz}\right), 119.5\left(\mathrm{C}-\mathrm{F},{ }^{1} J_{\mathrm{CF} 3}=272.7 \mathrm{~Hz}\right), 87.6\left(\mathrm{C}-\mathrm{F},{ }^{1} J_{\mathrm{CF}}=190.9 \mathrm{~Hz}\right), 85.7$ $\left(\mathrm{C}-\mathrm{F},{ }^{1} J_{\mathrm{CF}}=190.9 \mathrm{~Hz}\right), 84.1,48.5\left(\mathrm{C}-\mathrm{F},{ }^{2} J_{\mathrm{CF}}=27.3 \mathrm{~Hz}\right), 48.3\left(\mathrm{C}-\mathrm{F},{ }^{2} J_{\mathrm{CF}}=27.3 \mathrm{~Hz}\right), 28.3$. HRMS (APCI): $m / z[\mathrm{M}-\mathrm{Boc}+\mathrm{H}]^{+}$calcd. for $\mathrm{C}_{10} \mathrm{H}_{8} \mathrm{~F}_{4} \mathrm{NO} 234.0542$, found 234.0537.

tert-Butyl 6-chloro-3-fluoro-4-oxo-3,4-dihydroquinoline-1(2H)-carboxylate (2h). $260 \mathrm{mg}$ of tert-butyl 4-oxo-6-chloro-3,4-dihydroquinoline- $1(2 \mathrm{H})$-carboxylate $(0.92 \mathrm{mmol}, 1.0$ equiv) were used following the described procedure. Purification via flash column chromatog- 
raphy on silica gel (95:5 petroleum ether/ethyl acetate) yielded $107 \mathrm{mg}$ of $\mathbf{2 h}$ as a yellow solid (39\%). m.p. 117-121 ${ }^{\circ} \mathrm{C} .{ }^{1} \mathrm{H}-\mathrm{NMR}(400 \mathrm{MHz}$, Chloroform- $d) \delta 7.97(\mathrm{~d}, J=2.6 \mathrm{~Hz}, 1 \mathrm{H})$, $7.81(\mathrm{~d}, J=9.0 \mathrm{~Hz}, 1 \mathrm{H}), 7.48(\mathrm{dd}, J=9.0,2.6 \mathrm{~Hz}, 1 \mathrm{H}), 5.09(\mathrm{ddd}, J=46.8,10.4,4.7 \mathrm{~Hz}, 1 \mathrm{H})$, $4.60(\mathrm{td}, J=13.5,4.6 \mathrm{~Hz}, 1 \mathrm{H}), 4.06(\mathrm{ddd}, J=13.4,10.4,4.9 \mathrm{~Hz}, 1 \mathrm{H}), 1.56(\mathrm{~s}, 9 \mathrm{H}) .{ }^{19} \mathrm{~F}-\mathrm{NMR}$ (376 MHz, Chloroform-d) $\delta-198.92 .{ }^{13} \mathrm{C}-\mathrm{NMR}(101 \mathrm{MHz}$, Chloroform-d) $\delta 188.5$ (C-F, $\left.{ }^{2} J_{\mathrm{CF}}=15.2 \mathrm{~Hz}\right), 188.4\left(\mathrm{C}-\mathrm{F},{ }^{2} J_{\mathrm{CF}}=15.2 \mathrm{~Hz}\right), 152.51,142.5,134.8,130.4,127.4,125.3,124.1$, $87.8\left(\mathrm{C}-\mathrm{F},{ }^{1} J_{\mathrm{CF}}=191.9 \mathrm{~Hz}\right), 85.9\left(\mathrm{C}-\mathrm{F},{ }^{1} J_{\mathrm{CF}}=191.9 \mathrm{~Hz}\right), 83.6,48.6\left(\mathrm{C}-\mathrm{F},{ }^{2} J_{\mathrm{CF}}=26.3 \mathrm{~Hz}\right), 48.4$ $\left(\mathrm{C}-\mathrm{F},{ }^{2} J_{\mathrm{CF}}=26.3 \mathrm{~Hz}\right), 28.3$. HRMS (APCI): $\mathrm{m} / z[\mathrm{M}+\mathrm{H}]^{+}$calcd. for $\mathrm{C}_{14} \mathrm{H}_{16} \mathrm{ClFNO}_{3} 300.0797$, found 300.0799 .

tert-Butyl 6-bromo-3-fluoro-4-oxo-3,4-dihydroquinoline-1(2H)-carboxylate (2i). $380 \mathrm{mg}$ of tert-butyl 4-oxo-6-bromo-3,4-dihydroquinoline-1(2H)-carboxylate (1.16 mmol, 1.0 equiv) were used following the described procedure. Purification via flash column chromatography on silica gel (95:5 petroleum ether/ethyl acetate) yielded $165 \mathrm{mg}$ of $\mathbf{2} \mathbf{i}$ as a white solid (41\%). m.p. $111-114{ }^{\circ} \mathrm{C} .{ }^{1} \mathrm{H}-\mathrm{NMR}(400 \mathrm{MHz}$, Chloroform- $d) \delta 8.12(\mathrm{~d}, J=2.4 \mathrm{~Hz}, 1 \mathrm{H})$, $7.75(\mathrm{~d}, J=9.0 \mathrm{~Hz}, 1 \mathrm{H}), 7.62(\mathrm{dd}, J=9.0,2.5 \mathrm{~Hz}, 1 \mathrm{H}), 5.09(\mathrm{ddd}, J=46.8,10.4,4.7 \mathrm{~Hz}, 1 \mathrm{H})$, $4.59(\mathrm{td}, J=13.5,4.6 \mathrm{~Hz}, 1 \mathrm{H}), 4.05(\mathrm{ddd}, J=13.4,10.4,5.0 \mathrm{~Hz}, 1 \mathrm{H}), 1.56(\mathrm{~s}, 9 \mathrm{H}) .{ }^{19} \mathrm{~F}-\mathrm{NMR}$ (376 MHz, Chloroform-d) $\delta-198.90 .{ }^{13} \mathrm{C}-\mathrm{NMR}(101 \mathrm{MHz}$, Chloroform-d) $\delta 188.4$ (C-F, $\left.{ }^{2} J_{\mathrm{CF}}=15.2 \mathrm{~Hz}\right), 188.3\left(\mathrm{C}-\mathrm{F},{ }^{2} J_{\mathrm{CF}}=15.2 \mathrm{~Hz}\right), 152.4,143.0,137.7,130.5,125.5,124.4,117.8,87.8$ $\left(\mathrm{C}-\mathrm{F},{ }^{1} J_{\mathrm{CF}}=191.9 \mathrm{~Hz}\right), 85.9\left(\mathrm{C}-\mathrm{F},{ }^{1} J_{\mathrm{CF}}=191.9 \mathrm{~Hz}\right), 83.7,48.6\left(\mathrm{C}-\mathrm{F},{ }^{2} J_{\mathrm{CF}}=27.3 \mathrm{~Hz}\right), 48.3(\mathrm{C}-\mathrm{F}$, $\left.{ }^{2} J_{\mathrm{CF}}=27.3 \mathrm{~Hz}\right), 28.3$. HRMS (APCI): $m / z[\mathrm{M}-\mathrm{Boc}+\mathrm{H}]^{+}$calcd. for $\mathrm{C}_{9} \mathrm{H}_{8} \mathrm{BrFNO} 243.9773$, found 243.9768 .

tert-Butyl 3-fluoro-6-iodo-4-oxo-3,4-dihydroquinoline-1(2H)-carboxylate (2j). $339 \mathrm{mg}$ of tert-butyl 4-oxo-6-iodo-3,4-dihydroquinoline-1(2H)-carboxylate (0.91 mmol, 1.0 equiv) were used following the described procedure. Purification via flash column chromatography on silica gel (97:3 petroleum ether/ethyl acetate) yielded $160 \mathrm{mg}$ of $\mathbf{2} \mathbf{j}$ as a white solid (45\%). m.p. ${ }^{113-117}{ }^{\circ} \mathrm{C} .{ }^{1} \mathrm{H}-\mathrm{NMR}(400 \mathrm{MHz}$, Chloroform-d) $\delta 8.31(\mathrm{~d}, J=2.2 \mathrm{~Hz}, 1 \mathrm{H})$, $7.80(\mathrm{dd}, J=8.9,2.3 \mathrm{~Hz}, 1 \mathrm{H}), 7.61(\mathrm{~d}, J=8.9 \mathrm{~Hz}, 1 \mathrm{H}), 5.08(\mathrm{ddd}, J=46.8,10.4,4.7 \mathrm{~Hz}, 1 \mathrm{H})$, 4.59 (td, $J=13.5,4.7 \mathrm{~Hz}, 1 \mathrm{H}), 4.04(\mathrm{ddd}, J=13.4,10.4,5.0 \mathrm{~Hz}, 1 \mathrm{H}), 1.56(\mathrm{~s}, 9 \mathrm{H}) .{ }^{19} \mathrm{~F}-\mathrm{NMR}$ (376 MHz, Chloroform-d) $\delta-198.84 .{ }^{13} \mathrm{C}-\mathrm{NMR}(101 \mathrm{MHz}$, Chloroform-d) $\delta 188.3$ (C-F, $\left.{ }^{2} J_{\mathrm{CF}}=15.2 \mathrm{~Hz}\right), 188.2\left(\mathrm{C}-\mathrm{F},{ }^{2} J_{\mathrm{CF}}=15.2 \mathrm{~Hz}\right), 152.4,143.6,143.4,136.7,125.6,124.5,88.0,87.6$ $\left(\mathrm{C}-\mathrm{F},{ }^{1} J_{\mathrm{CF}}=190.9 \mathrm{~Hz}\right), 85.8\left(\mathrm{C}-\mathrm{F},{ }^{1} J_{\mathrm{CF}}=190.9 \mathrm{~Hz}\right), 83.7,48.5\left(\mathrm{C}-\mathrm{F},{ }^{2} J_{\mathrm{CF}}=24.2 \mathrm{~Hz}\right), 48.3(\mathrm{C}-\mathrm{F}$, $\left.{ }^{2} J_{\mathrm{CF}}=24.2 \mathrm{~Hz}\right), 28.3$. HRMS (APCI): $m / z[\mathrm{M}+\mathrm{H}]^{+}$calcd. for $\mathrm{C}_{14} \mathrm{H}_{16} \mathrm{FINO}_{3} 392.0153$, found 392.0155 .

\subsection{General Procedure for the ATH of tert-Butyl 3-fluoro-4-oxo-3,4-dihydroquinoline- 1(2H)-carboxylates}

In a round-bottom tube charged with the corresponding 3-fluoro-4-oxo-3,4dihydroquinoline-1 $(2 H)$-carboxylate (1.0 equiv) set under argon, the necessary volume of a solution of $(R, R)-\mathrm{A}$ in acetonitrile $(1.0 \mathrm{~mL}$ of a $1.24 \mathrm{mg} / \mathrm{mL}$ solution; 0.005 equiv) was added. The mixture was stirred for one minute before adding, by syringe, the necessary volume of formic acid/triethylamine (1:1) mixture (6.0 equiv). The reaction mixture was stirred at $40{ }^{\circ} \mathrm{C}$ for the time needed and then quenched with $3 \mathrm{~mL}$ of saturated $\mathrm{NaHCO}_{3}$ aqueous solution. The media was extracted with $\mathrm{CH}_{2} \mathrm{Cl}_{2}(2 \times 4 \mathrm{~mL})$ and the organic layers were dried over $\mathrm{MgSO}_{4}$, filtered, and concentrated under vacuum. The diastereoisomeric ratio was determined by ${ }^{1} \mathrm{H}-\mathrm{NMR}$ analysis of the crude product. The product was purified with a flash column chromatography on silica gel (petroleum ether/EtOAc) and the enantiomeric excess was determined by SFC analysis (CHIRALPAK IE or IF column) (see Supplementary Materials for NMR and SFC spectra).

tert-Butyl (3R,4S)-3-fluoro-4-hydroxy-3,4-dihydroquinoline-1(2H)-carboxylate (3a). $100 \mathrm{mg}$ of tert-butyl 3-fluoro-4-oxo-3,4-dihydroquinoline-1(2H)-carboxylate (2a) (0.38 mmol; 1.0 equiv) were used according to the described procedure (reaction time of $3 \mathrm{~h}$ ). The product was purified by flash column chromatography on silica gel (petroleum ether/ethyl acetate 75:25) and yielded $93 \mathrm{mg}$ of a beige/white solid (93\%). m.p. $142-144{ }^{\circ} \mathrm{C} \mathrm{dr}$ (cis/trans) = 98:2, 
$\mathrm{ee}_{\text {cis }}=>99 \% .[\alpha]_{\mathrm{D}}{ }^{30}=-5\left(\mathrm{c} 1.0, \mathrm{CHCl}_{3}\right) .{ }^{1} \mathrm{H}-\mathrm{NMR}(400 \mathrm{MHz}$, Chloroform- $d) \delta 7.70(\mathrm{~d}$, $J=8.3 \mathrm{~Hz}, 1 \mathrm{H}), 7.51(\mathrm{dt}, J=7.6,1.4 \mathrm{~Hz}, 1 \mathrm{H}), 7.34-7.24(\mathrm{~m}, 1 \mathrm{H}), 7.14(\mathrm{td}, J=7.5,1.2 \mathrm{~Hz}$, $1 \mathrm{H}), 5.05(\mathrm{ddt}, J=50.8,4.8,3.5 \mathrm{~Hz}, 1 \mathrm{H}), 4.78(\mathrm{dd}, J=21.5,5.9 \mathrm{~Hz}, 1 \mathrm{H}), 4.10(\mathrm{ddd}, J=14.9$, $14.2,4.8 \mathrm{~Hz}, 1 \mathrm{H}), 3.94(\mathrm{ddd}, J=29.9,14.2,3.7 \mathrm{~Hz}, 1 \mathrm{H}), 2.38(\mathrm{~d}, J=8.3 \mathrm{~Hz}, 1 \mathrm{H}), 1.53(\mathrm{~s}, 9 \mathrm{H})$. ${ }^{19}$ F-NMR (376 MHz, Chloroform- $d$ ) $\delta-200.34 .{ }^{13} \mathrm{C}-\mathrm{NMR}(101 \mathrm{MHz}$, Chloroform- $d$ ) $\delta 153.8$, $136.8,128.2,127.9,127.3,124.3,123.5,89.6\left(\mathrm{C}-\mathrm{F},{ }^{1} J_{\mathrm{CF}}=176.8 \mathrm{~Hz}\right), 87.9\left(\mathrm{C}-\mathrm{F},{ }^{1} J_{\mathrm{CF}}=176.8 \mathrm{~Hz}\right)$, $81.9,68.0\left(\mathrm{C}-\mathrm{F},{ }^{2} J_{\mathrm{CF}}=19.2 \mathrm{~Hz}\right), 67.8\left(\mathrm{C}-\mathrm{F},{ }^{2} J_{\mathrm{CF}}=19.2 \mathrm{~Hz}\right), 46.3\left(\mathrm{C}-\mathrm{F},{ }^{2} J_{\mathrm{CF}}=23.2 \mathrm{~Hz}\right), 46.1(\mathrm{C}-\mathrm{F}$, ${ }^{2} J_{\mathrm{CF}}=23.2 \mathrm{~Hz}$ ), 28.4. HRMS (APCI): $m / z[\mathrm{M}+\mathrm{H}]^{+}$calcd. for $\mathrm{C}_{14} \mathrm{H}_{19} \mathrm{FNO}_{3} 268.1343$, found 268.1345. SFC: Chiralpak IE, scCO $/ \mathrm{EtOH} \mathrm{96/4,} 2.0 \mathrm{~mL} / \mathrm{min}, \mathrm{P}=100 \mathrm{bar}, \lambda=215 \mathrm{~nm}$, $\mathrm{t}_{\mathrm{R}}[$ trans $]=11.04 \mathrm{~min}, \mathrm{t}_{\mathrm{R}}[$ trans $]=12.85 \mathrm{~min}, \mathrm{t}_{\mathrm{R}}[$ cis- $(3 R, 4 \mathrm{~S})]=14.54 \mathrm{~min}$ (major), $\mathrm{t}_{\mathrm{R}}[$ cis$(3 S, 4 R)]=15.43 \mathrm{~min}$.

tert-Butyl (3R,4S)-3-fluoro-4-hydroxy-6-methyl-3,4-dihydroquinoline-1(2H)-carboxylate (3b). $100 \mathrm{mg}$ of tert-butyl 3-fluoro-6-methyl-4-oxo-3,4-dihydroquinoline-1(2H)-carboxylate (2b) ( $0.36 \mathrm{mmol} ; 1.0$ equiv) were used according to the described procedure (reaction time of $3 \mathrm{~h}$ ). The product was purified by flash column chromatography on silica gel (petroleum ether/ethyl acetate 75:25) and yielded $91 \mathrm{mg}$ of a colourless oil (91\%). dr (cis/trans) = 98:2, $\mathrm{ee}_{\text {cis }}=>99 \% .[\alpha]_{\mathrm{D}}{ }^{22}=-8.4\left(c 1.0, \mathrm{CHCl}_{3}\right) .{ }^{1} \mathrm{H}-\mathrm{NMR}(400 \mathrm{MHz}$, Chloroform- $d) \delta 7.56$ $(\mathrm{d}, J=8.4 \mathrm{~Hz}, 1 \mathrm{H}), 7.34-7.28(\mathrm{~m}, 1 \mathrm{H}), 7.08(\mathrm{dd}, J=8.4,2.1 \mathrm{~Hz}, 1 \mathrm{H}), 5.00(\mathrm{dq}, J=50.9$, $4.2 \mathrm{~Hz}, 1 \mathrm{H}), 4.72(\mathrm{dd}, J=22.0,7.0 \mathrm{~Hz}, 1 \mathrm{H}), 4.10-3.84(\mathrm{~m}, 2 \mathrm{H}), 2.53(\mathrm{~s}, 1 \mathrm{H}), 2.32$ (s, 3H), 1.52 (s, 9H). ${ }^{19} \mathrm{~F}-\mathrm{NMR}(376 \mathrm{MHz}$, Chloroform- $d) \delta-200.17 .{ }^{13} \mathrm{C}-\mathrm{NMR}(101 \mathrm{MHz}$, Chloroform-d) $\delta 153.8,134.2,133.9,128.9,127.6,123.4,89.7,88.0\left(\mathrm{C}-\mathrm{F},{ }^{1} J_{\mathrm{CF}}=176.8 \mathrm{~Hz}\right)$, $81.7\left(\mathrm{C}-\mathrm{F},{ }^{1} J_{\mathrm{CF}}=176.8 \mathrm{~Hz}\right), 67.9\left(\mathrm{C}-\mathrm{F},{ }^{2} J_{\mathrm{CF}}=20.2 \mathrm{~Hz}\right), 67.7\left(\mathrm{C}-\mathrm{F},{ }^{2} J_{\mathrm{CF}}=20.2 \mathrm{~Hz}\right), 46.2(\mathrm{C}-\mathrm{F}$, $\left.{ }^{2} J_{\mathrm{CF}}=23.2 \mathrm{~Hz}\right), 46.0\left(\mathrm{C}-\mathrm{F},{ }^{2} J_{\mathrm{CF}}=23.2 \mathrm{~Hz}\right), 28.4,20.9$. HRMS (APCI): $\mathrm{m} / z[\mathrm{M}+\mathrm{H}]^{+}$calcd. for $\mathrm{C}_{15} \mathrm{H}_{21} \mathrm{FNO}_{3}$ 282.1500, found 282.1502. SFC: Chiralpak IE, scCO $/ \mathrm{EtOH} 97.5 / 2.5,2.0$ $\mathrm{mL} / \mathrm{min}, \mathrm{P}=100$ bar, $\lambda=215 \mathrm{~nm}, \mathrm{t}_{\mathrm{R}}[$ trans $]=27.71 \mathrm{~min}, \mathrm{t}_{\mathrm{R}}[$ cis- $(3 R, 4 \mathrm{~S})]=32.43 \mathrm{~min}$ (major), $\mathrm{t}_{\mathrm{R}}[$ cis- $(3 S, 4 R)]=37.48 \mathrm{~min}, \mathrm{t}_{\mathrm{R}}[$ trans $]=42.41 \mathrm{~min}$.

tert-Butyl $(3 R, 4 S)$-3-fluoro-4-hydroxy-6-methoxy-3,4-dihydroquinoline-1(2H)-carboxylate (3c). $100 \mathrm{mg}$ of tert-butyl 3-fluoro-6-methoxy-4-oxo-3,4-dihydroquinoline-1(2H)-carboxylate (2c) $(0.34 \mathrm{mmol} ; 1.0$ equiv) were used according to the described procedure (reaction time of $5 \mathrm{~h}$ ). The product was purified by flash column chromatography on silica gel (petroleum ether/ethyl acetate 75:25) and yielded $95 \mathrm{mg}$ of a white/red solid (95\%). m.p. $114-118{ }^{\circ} \mathrm{C}$ $\mathrm{dr}($ cis $/$ trans $)=98: 2, \mathrm{ee}_{\text {cis }}=>99 \% .[\alpha]_{\mathrm{D}}{ }^{22}=-13.3\left(\right.$ c 1.0, $\left.\mathrm{CHCl}_{3}\right) .{ }^{1} \mathrm{H}-\mathrm{NMR}(400 \mathrm{MHz}$, Chloroform- $d) \delta 7.57(\mathrm{~d}, J=9.1 \mathrm{~Hz}, 1 \mathrm{H}), 7.05(\mathrm{dd}, J=3.0,1.0 \mathrm{~Hz}, 1 \mathrm{H}), 6.83(\mathrm{dd}, J=9.1,3.0 \mathrm{~Hz}$, $1 \mathrm{H}), 5.06(\mathrm{dd}, J=51.1,3.7 \mathrm{~Hz}, 1 \mathrm{H}), 4.74(\mathrm{ddd}, J=22.7,9.4,3.2 \mathrm{~Hz}, 1 \mathrm{H}), 4.08(\mathrm{ddd}, J=15.9$, 14.4, $4.4 \mathrm{~Hz}, 1 \mathrm{H}), 3.92(\mathrm{ddd}, J=31.4,14.5,3.7 \mathrm{~Hz}, 1 \mathrm{H}), 3.81(\mathrm{~s}, 3 \mathrm{H}), 2.37$ (d, J = 9.4 Hz, 1H), 1.52 (s, 9H). ${ }^{19}$ F-NMR (376 MHz, Chloroform-d) $\delta-200.32 .{ }^{13} \mathrm{C}-\mathrm{NMR}(101 \mathrm{MHz}$, Chloroform- $d$ ) $\delta 156.5,153.9,129.8,129.4,125.0,114.3,111.4,89.9\left(\mathrm{C}-\mathrm{F},{ }^{1} J_{\mathrm{CF}}=176.8 \mathrm{~Hz}\right), 88.1$ $\left(\mathrm{C}-\mathrm{F},{ }^{1} J_{\mathrm{CF}}=176.8 \mathrm{~Hz}\right), 81.6,68.2\left(\mathrm{C}-\mathrm{F},{ }^{2} J_{\mathrm{CF}}=20.2 \mathrm{~Hz}\right), 68.0\left(\mathrm{C}-\mathrm{F},{ }^{2} J_{\mathrm{CF}}=20.2 \mathrm{~Hz}\right), 55.7,46.6$ $\left(\mathrm{C}-\mathrm{F},{ }^{2} J_{\mathrm{CF}}=23.2 \mathrm{~Hz}\right), 46.3\left(\mathrm{C}-\mathrm{F},{ }^{2} J_{\mathrm{CF}}=23.2 \mathrm{~Hz}\right), 28.4$. HRMS $(\mathrm{APCI}): \mathrm{m} / z[\mathrm{M}+\mathrm{Na}]^{+}$calcd. for $\mathrm{C}_{15} \mathrm{H}_{20} \mathrm{FNO}_{4} \mathrm{Na} 320.1269$, found 320.1273. SFC: Chiralpak IF, scCO $2 / \mathrm{EtOH} 95.5 / 45,2.0$ $\mathrm{mL} / \mathrm{min}, \mathrm{P}=100$ bar, $\lambda=254 \mathrm{~nm}, \mathrm{t}_{\mathrm{R}}[$ trans $]=15.71 \mathrm{~min}, \mathrm{t}_{\mathrm{R}}[$ cis- $(3 S, 4 R)]=17.11 \mathrm{~min}, \mathrm{t}_{\mathrm{R}}$ $[$ trans $]=18.69 \mathrm{~min}, \mathrm{t}_{\mathrm{R}}[$ cis- $(3 R, 4 \mathrm{~S})]=22.79 \mathrm{~min}$ (major)

tert-Butyl $(3 R, 4 S)$-3-fluoro-4-hydroxy-7-methoxy-3,4-dihydroquinoline-1(2H)-carboxylate (3d). $100 \mathrm{mg}$ of tert-butyl 3-fluoro-7-methoxy-4-oxo-3,4-dihydroquinoline-1(2H)-carboxylate (2d) $(0.34 \mathrm{mmol} ; 1.0$ equiv) were used according to the described procedure (reaction time of $5 \mathrm{~h}$ ). The product was purified by flash column chromatography on silica gel (petroleum ether/ethyl acetate 75:25) and yielded $95 \mathrm{mg}$ of a beige/white solid (95\%). m.p. $92-96{ }^{\circ} \mathrm{C}$ $\mathrm{dr}($ cis $/$ trans $)=95: 5, \mathrm{ee}_{\text {cis }}=>99 \% . \quad[\alpha]_{\mathrm{D}}{ }^{22}=-9.2\left(\right.$ c $\left.1.0, \mathrm{CHCl}_{3}\right) .{ }^{1} \mathrm{H}-\mathrm{NMR}(400 \mathrm{MHz}$, Chloroform-d) $\delta 7.42-7.31(\mathrm{~m}, 2 \mathrm{H}), 6.70(\mathrm{dt}, J=8.6,2.3 \mathrm{~Hz}, 1 \mathrm{H}), 4.98(\mathrm{dd}, J=50.0,5.9 \mathrm{~Hz}$, $1 \mathrm{H}), 4.76(\mathrm{~d}, J=19.1 \mathrm{~Hz}, 1 \mathrm{H}), 4.16-4.04(\mathrm{~m}, 1 \mathrm{H}), 4.01-3.85(\mathrm{~m}, 1 \mathrm{H}), 3.80(\mathrm{~s}, 3 \mathrm{H}), 2.33$ (s, 1H), 1.54 (s, 9H). ${ }^{19}$ F-NMR (376 MHz, Chloroform-d) $\delta-201.08 .{ }^{13} \mathrm{C}-\mathrm{NMR}(101 \mathrm{MHz}$, Chloroform- $d$ ) $\delta 159.7,153.6,138.0,128.9,119.8,110.8,108.6,89.1\left(\mathrm{C}-\mathrm{F},{ }^{1} J_{\mathrm{CF}}=177.8 \mathrm{~Hz}\right)$, $87.3\left(\mathrm{C}-\mathrm{F},{ }^{1} J_{\mathrm{CF}}=177.8 \mathrm{~Hz}\right), 82.0,67.6\left(\mathrm{C}-\mathrm{F},{ }^{2} J_{\mathrm{CF}}=19.2 \mathrm{~Hz}\right), 67.4\left(\mathrm{C}-\mathrm{F},{ }^{2} J_{\mathrm{CF}}=19.2 \mathrm{~Hz}\right), 55.5$, 
$45.7\left(\mathrm{C}-\mathrm{F},{ }^{2} J_{\mathrm{CF}}=25.3 \mathrm{~Hz}\right), 45.5\left(\mathrm{C}-\mathrm{F},{ }^{2} J_{\mathrm{CF}}=25.3 \mathrm{~Hz}\right), 28.5$. HRMS (APCI): $m / z\left[\mathrm{M}+\mathrm{NH}_{4}\right]^{+}$ calcd. for $\mathrm{C}_{15} \mathrm{H}_{24} \mathrm{FN}_{2} \mathrm{O}_{4} 315.1715$, found 315.1718. SFC: Chiralpak IE, scCO $2 / \mathrm{EtOH} 95 / 5$, $2.0 \mathrm{~mL} / \mathrm{min}, \mathrm{P}=100$ bar, $\lambda=215 \mathrm{~nm}, \mathrm{t}_{\mathrm{R}}[$ trans $]=11.87 \mathrm{~min}, \mathrm{t}_{\mathrm{R}}[$ trans $]=13.19 \mathrm{~min}, \mathrm{t}_{\mathrm{R}}$ $[$ cis- $(3 R, 4 S)]=16.63 \mathrm{~min}$ (major), $\mathrm{t}_{\mathrm{R}}[\operatorname{cis}-(3 S, 4 R)]=18.62 \mathrm{~min}$.

tert-Butyl (3R,4S)-3-fluoro-4-hydroxy-6,7-dimethoxy-3,4-dihydroquinoline-1(2H)-carboxylate (3e). $100 \mathrm{mg}$ of tert-butyl 3-fluoro-6,7-dimethoxy-4-oxo-3,4-dihydroquinoline-1(2H)-carboxylate (2e) $(0.31 \mathrm{mmol} ; 1.0$ equiv) were used according to the described procedure with $1.0 \mathrm{~mol} \%$ of catalyst $(R, R)$-D (reaction time of $24 \mathrm{~h}$ ). The product was purified by flash column chromatography on silica gel (petroleum ether/ethyl acetate 60:40) and yielded $90 \mathrm{mg}$ of a colourless oil $(90 \%)$. dr $($ cis $/$ trans $)=94: 6$, ee $_{\text {cis }}=99 \% .[\alpha]_{\mathrm{D}}{ }^{22}=-11.8\left(\right.$ c 1.0, $\left.\mathrm{CHCl}_{3}\right) .{ }^{1} \mathrm{H}-\mathrm{NMR}$ $(400 \mathrm{MHz}$, Chloroform-d) $\delta 7.32(\mathrm{~s}, 1 \mathrm{H}), 6.97(\mathrm{~d}, J=0.8 \mathrm{~Hz}, 1 \mathrm{H}), 4.99$ (ddt, $J=50.2,5.6,3.4 \mathrm{~Hz}$, $1 \mathrm{H}), 4.74(\mathrm{dq}, J=19.9,3.5 \mathrm{~Hz}, 1 \mathrm{H}), 4.20-4.11(\mathrm{~m}, 1 \mathrm{H}), 3.94-3.80(\mathrm{~m}, 7 \mathrm{H}), 2.38(\mathrm{~d}, J=8.4 \mathrm{~Hz}$, 1H), 1.53 (s, 9H). ${ }^{19}$ F-NMR (376 MHz, Chloroform-d) $\delta-202.03 .{ }^{13} \mathrm{C}-\mathrm{NMR}(101 \mathrm{MHz}$, Chloroform- $d$ ) $\delta 153.8,148.5,146.2,130.4,119.6,110.0,107.5,89.1\left(\mathrm{C}-\mathrm{F},{ }^{1} J_{\mathrm{CF}}=177.8 \mathrm{~Hz}\right), 87.3$ $\left(\mathrm{C}-\mathrm{F},{ }^{1} J_{\mathrm{CF}}=177.8 \mathrm{~Hz}\right), 81.7,67.7\left(\mathrm{C}-\mathrm{F},{ }^{2} J_{\mathrm{CF}}=20.2 \mathrm{~Hz}\right), 67.5\left(\mathrm{C}-\mathrm{F},{ }^{2} J_{\mathrm{CF}}=20.2 \mathrm{~Hz}\right), 56.2,56.1$, $46.0\left(\mathrm{C}-\mathrm{F},{ }^{2} J_{\mathrm{CF}}=24.2 \mathrm{~Hz}\right), 45.8\left(\mathrm{C}-\mathrm{F},{ }^{2} J_{\mathrm{CF}}=24.2 \mathrm{~Hz}\right), 28.5$. HRMS (APCI): $m / z[\mathrm{M}+\mathrm{Na}]^{+}$ calcd. for $\mathrm{C}_{16} \mathrm{H}_{22} \mathrm{FNO}_{5} \mathrm{Na} 350.1374$, found 320.1377. SFC: Chiralpak IF, $s c \mathrm{CO}_{2} / \mathrm{EtOH} 95 / 5$, $2.0 \mathrm{~mL} / \mathrm{min}, \mathrm{P}=100$ bar, $\lambda=215 \mathrm{~nm}, \mathrm{t}_{\mathrm{R}}$ [trans $]=14.75 \mathrm{~min}, \mathrm{t}_{\mathrm{R}}[$ trans $]=15.89 \mathrm{~min}, \mathrm{t}_{\mathrm{R}}$ $[$ cis- $(3 R, 4 S)]=17.45 \mathrm{~min}$ (major), $\mathrm{t}_{\mathrm{R}}[$ cis- $(3 S, 4 R)]=19.07 \mathrm{~min}$.

tert-Butyl (3R,4S)-3,6-difluoro-4-hydroxy-3,4-dihydroquinoline-1(2H)-carboxylate (3f). $100 \mathrm{mg}$ of tert-butyl 3,6-difluoro-4-oxo-3,4-dihydroquinoline-1(2H)-carboxylate (2f) $(0.35 \mathrm{mmol}$; 1.0 equiv) were used according to the described procedure (reaction time of $24 \mathrm{~h}$ ). The product was purified by flash column chromatography on silica gel (petroleum ether/ethyl acetate 75:25) and yielded $70 \mathrm{mg}$ of a white solid (70\%). m.p. $78-82{ }^{\circ} \mathrm{C} \mathrm{dr}($ cis $/$ trans $)=98: 2$, $\mathrm{ee}_{\text {cis }}=>99 \% .[\alpha]_{\mathrm{D}}{ }^{22}=-11.7\left(c 1.0, \mathrm{CHCl}_{3}\right) .{ }^{1} \mathrm{H}-\mathrm{NMR}(400 \mathrm{MHz}$, Chloroform- $d) \delta 7.64$ $(\mathrm{dd}, J=9.3,5.1 \mathrm{~Hz}, 1 \mathrm{H}), 7.23(\mathrm{ddd}, J=8.8,3.0,1.0 \mathrm{~Hz}, 1 \mathrm{H}), 6.97(\mathrm{td}, J=8.9,8.1,3.1 \mathrm{~Hz}$, $1 \mathrm{H}), 5.08(\mathrm{dq}, J=51.2,3.6 \mathrm{~Hz}, 1 \mathrm{H}), 4.71(\mathrm{ddd}, J=23.6,9.5,3.1 \mathrm{~Hz}, 1 \mathrm{H}), 4.09(\mathrm{ddd}, J=16.6$, 14.6, 3.9 Hz, 1H), $3.91(\mathrm{ddd}, J=33.1,14.6,3.6 \mathrm{~Hz}, 1 \mathrm{H}), 2.48(\mathrm{dd}, J=9.7,2.0 \mathrm{~Hz}, 1 \mathrm{H}), 1.52$ $(\mathrm{s}, 9 \mathrm{H}) .{ }^{19} \mathrm{~F}-\mathrm{NMR}\left(376 \mathrm{MHz}\right.$, Chloroform-d) $\delta-118.05,-200.38 .{ }^{13} \mathrm{C}-\mathrm{NMR}(101 \mathrm{MHz}$, Chloroform-d) $\delta 160.8\left(\mathrm{C}-\mathrm{F},{ }^{1} J_{\mathrm{CF}}=244.4 \mathrm{~Hz}\right), 158.4\left(\mathrm{C}-\mathrm{F},{ }^{1} J_{\mathrm{CF}}=244.4 \mathrm{~Hz}\right), 153.7,132.6$ $\left(\mathrm{C}-\mathrm{F},{ }^{4} J_{\mathrm{CF}}=2.0 \mathrm{~Hz}\right), 132.6\left(\mathrm{C}-\mathrm{F},{ }^{4} J_{\mathrm{CF}}=2.0 \mathrm{~Hz}\right), 130.36\left(\mathrm{C}-\mathrm{F},{ }^{3} J_{\mathrm{CF}}=6.1 \mathrm{~Hz}\right), 130.3(\mathrm{C}-\mathrm{F}$, $\left.{ }^{3} J_{\mathrm{CF}}=6.1 \mathrm{~Hz}\right), 125.4\left(\mathrm{C}-\mathrm{F},{ }^{3} J_{\mathrm{CF}}=7.1 \mathrm{~Hz}\right), 125.3\left(\mathrm{C}-\mathrm{F},{ }^{3} J_{\mathrm{CF}}=7.1 \mathrm{~Hz}\right), 115.1\left(\mathrm{C}-\mathrm{F},{ }^{2} J_{\mathrm{CF}}=22.2 \mathrm{~Hz}\right)$, $114.9\left(\mathrm{C}-\mathrm{F},{ }^{2} J_{\mathrm{CF}}=22.2 \mathrm{~Hz}\right), 113.5\left(\mathrm{C}-\mathrm{F},{ }^{2} J_{\mathrm{CF}}=22.2 \mathrm{~Hz}\right), 113.3\left(\mathrm{C}-\mathrm{F},{ }^{2} J_{\mathrm{CF}}=22.2 \mathrm{~Hz}\right), 89.7$ $\left(\mathrm{C}-\mathrm{F},{ }^{1} J_{\mathrm{CF}}=176.8 \mathrm{~Hz}\right), 87.9\left(\mathrm{C}-\mathrm{F},{ }^{1} J_{\mathrm{CF}}=176.8 \mathrm{~Hz}\right), 82.1,67.9\left(\mathrm{C}-\mathrm{F},{ }^{2} J_{\mathrm{CF}}=20.2 \mathrm{~Hz}\right), 67.7$ $\left(\mathrm{C}-\mathrm{F},{ }^{2} J_{\mathrm{CF}}=20.2 \mathrm{~Hz}\right), 46.8\left(\mathrm{C}-\mathrm{F},{ }^{2} J_{\mathrm{CF}}=23.2 \mathrm{~Hz}\right), 46.6\left(\mathrm{C}-\mathrm{F},{ }^{2} J_{\mathrm{CF}}=23.2 \mathrm{~Hz}\right), 28.4$. HRMS (APCI): $m / z[\mathrm{M}+\mathrm{H}]^{+}$calcd. for $\mathrm{C}_{14} \mathrm{H}_{18} \mathrm{~F}_{2} \mathrm{NO}_{3} 286.1249$, found 282.1248. SFC: Chiralpak IE, scCO $2 / \mathrm{MeOH} 94 / 6,2.0 \mathrm{~mL} / \mathrm{min}, \mathrm{P}=100 \mathrm{bar}, \lambda=215 \mathrm{~nm}, \mathrm{t}_{\mathrm{R}}[$ trans $]=4.50 \mathrm{~min}, \mathrm{t}_{\mathrm{R}}$ $[$ trans $]=4.96 \mathrm{~min}, \mathrm{t}_{\mathrm{R}}[$ cis- $(3 S, 4 R)]=6.44 \mathrm{~min}, \mathrm{t}_{\mathrm{R}}[$ cis- $(3 R, 4 S)]=7.00 \mathrm{~min}$ (major).

tert-Butyl (3R,4S)-3-fluoro-4-hydroxy-6-(trifluoromethyl)-3,4-dihydroquinoline-1(2H)carboxylate (3g). $100 \mathrm{mg}$ of tert-butyl 3-fluoro-6-trifluoromethyl-4-oxo-3,4-dihydroquinoline$1(2 \mathrm{H})$-carboxylate $(\mathbf{2 g})(0.30 \mathrm{mmol} ; 1.0$ equiv) were used according to the described procedure (reaction time of $3 \mathrm{~h}$ ). The product was purified by flash column chromatography on silica gel (petroleum ether/ethyl acetate 75:25) and yielded $97 \mathrm{mg}$ of a beige/white solid (97\%). m.p. $152-156{ }^{\circ} \mathrm{C}$ dr (cis/trans) $=98: 2$, ee $_{\text {cis }}=>99 \%$. $[\alpha]_{\mathrm{D}}{ }^{22}=-0.9($ c 1.0 , $\left.\mathrm{CHCl}_{3}\right) .{ }^{1} \mathrm{H}-\mathrm{NMR}(400 \mathrm{MHz}$, Chloroform-d) $\delta 7.88(\mathrm{~d}, J=8.7 \mathrm{~Hz}, 1 \mathrm{H}), 7.81(\mathrm{~d}, J=2.2 \mathrm{~Hz}$, $1 \mathrm{H}), 7.51(\mathrm{ddt}, J=8.7,2.2,0.7 \mathrm{~Hz}, 1 \mathrm{H}), 5.10(\mathrm{dddd}, J=50.9,7.7,3.7,1.4 \mathrm{~Hz}, 1 \mathrm{H}), 4.79$ (dd, $J=23.1,6.9 \mathrm{~Hz}, 1 \mathrm{H}), 4.17(\mathrm{tdd}, J=15.5,4.1,1.1 \mathrm{~Hz}, 1 \mathrm{H}), 3.91(\mathrm{dd}, J=33.7,3.6 \mathrm{~Hz}, 1 \mathrm{H})$, 2.45 (s, 1H), 1.54 (s, 9H). ${ }^{19}$ F-NMR (376 MHz, Chloroform-d) $\delta-62.20,-200.84 .{ }^{13}$ C-NMR $\left(101 \mathrm{MHz}\right.$, Chloroform-d) $\delta 153.4,139.7,128.3\left(\mathrm{C}-\mathrm{F},{ }^{1} J_{\mathrm{CF} 3}=272.7 \mathrm{~Hz}\right), 128.2,128.1,126.5$ $\left(\mathrm{C}-\mathrm{F},{ }^{2} J_{\mathrm{CF} 3}=33.3 \mathrm{~Hz}\right), 126.2\left(\mathrm{C}-\mathrm{F},{ }^{2} J_{\mathrm{CF} 3}=33.3 \mathrm{~Hz}\right), 125.9\left(\mathrm{C}-\mathrm{F},{ }^{2} J_{\mathrm{CF} 3}=33.3 \mathrm{~Hz}\right), 125.6(\mathrm{C}-\mathrm{F}$, $\left.{ }^{1} J_{\mathrm{CF} 3}=272.7 \mathrm{~Hz}+\mathrm{C}-\mathrm{F},{ }^{2} J_{\mathrm{CF} 3}=33.3 \mathrm{~Hz}\right), 125.1\left(\mathrm{C}-\mathrm{F},{ }^{3} J_{\mathrm{CF} 3}=4.0 \mathrm{~Hz}\right), 125.1\left(\mathrm{C}-\mathrm{F},{ }^{3} J_{\mathrm{CF} 3}=4.0 \mathrm{~Hz}\right)$, $124.4\left(\mathrm{C}-\mathrm{F},{ }^{3} J_{\mathrm{CF} 3}=4.0 \mathrm{~Hz}\right), 124.4\left(\mathrm{C}-\mathrm{F},{ }^{3} J_{\mathrm{CF} 3}=4.0 \mathrm{~Hz}\right), 123.4,122.9\left(\mathrm{C}-\mathrm{F},{ }^{1} J_{\mathrm{CF} 3}=272.7 \mathrm{~Hz}\right)$, $120.2\left(\mathrm{C}-\mathrm{F},{ }^{1} J_{\mathrm{CF} 3}=272.7 \mathrm{~Hz}\right), 89.1\left(\mathrm{C}-\mathrm{F},{ }^{1} J_{\mathrm{CF}}=176.8 \mathrm{~Hz}\right), 87.4\left(\mathrm{C}-\mathrm{F},{ }^{1} J_{\mathrm{CF}}=176.8 \mathrm{~Hz}\right), 82.7$, 
$67.7\left(\mathrm{C}-\mathrm{F},{ }^{2} J_{\mathrm{CF}}=20.2 \mathrm{~Hz}\right), 67.5\left(\mathrm{C}-\mathrm{F},{ }^{2} J_{\mathrm{CF}}=20.2 \mathrm{~Hz}\right), 46.8\left(\mathrm{C}-\mathrm{F},{ }^{2} J_{\mathrm{CF}}=22.2 \mathrm{~Hz}\right), 46.6(\mathrm{C}-\mathrm{F}$, ${ }^{2} J_{\mathrm{CF}}=22.2 \mathrm{~Hz}$ ), 28.3. HRMS (APCI): $m / z\left[\mathrm{M}+\mathrm{NH}_{4}\right]^{+}$calcd. for $\mathrm{C}_{15} \mathrm{H}_{21} \mathrm{FN}_{2} \mathrm{O} 3353.1483$, found 353.1483. SFC: Chiralpak IF, $s c \mathrm{CO}_{2} / \mathrm{EtOH} 96 / 4,2.0 \mathrm{~mL} / \mathrm{min}, \mathrm{P}=100 \mathrm{bar}, \lambda=254 \mathrm{~nm}$, $\mathrm{t}_{\mathrm{R}}[$ cis- $(3 R, 4 \mathrm{~S})]=5.68 \mathrm{~min}$ (major), $\mathrm{t}_{\mathrm{R}}[$ cis- $(3 S, 4 R)]=6.84 \mathrm{~min}, \mathrm{t}_{\mathrm{R}}[$ trans $]=8.54 \mathrm{~min}$.

tert-Butyl (3R,4S)-6-chloro-3-fluoro-4-hydroxy-3,4-dihydroquinoline-1(2H)-carboxylate (3h). $60 \mathrm{mg}$ of tert-butyl 3-fluoro-6-chloro-4-oxo-3,4-dihydroquinoline-1 $(2 \mathrm{H})$-carboxylate (2h) $(0.20 \mathrm{mmol} ; 1.0$ equiv) were used according to the described procedure (reaction time of $3 \mathrm{~h}$ ). The product was purified by flash column chromatography on silica gel (petroleum ether/ethyl acetate 75:25) and yielded $97 \mathrm{mg}$ of a beige/white solid (96\%). m.p. 113-116 ${ }^{\circ} \mathrm{C}$ $\mathrm{dr}($ cis $/$ trans $)=99: 1, \mathrm{ee}_{\text {cis }}=>99 \% .[\alpha]_{\mathrm{D}}{ }^{22}=-12.8\left(c 1.0, \mathrm{CHCl}_{3}\right) .{ }^{1} \mathrm{H}-\mathrm{NMR}(400 \mathrm{MHz}$, Chloroform- $d) \delta 7.66(\mathrm{~d}, J=8.9 \mathrm{~Hz}, 1 \mathrm{H}), 7.51(\mathrm{dd}, J=2.6,1.1 \mathrm{~Hz}, 1 \mathrm{H}), 7.22(\mathrm{~d}, J=9.2 \mathrm{~Hz}$, $1 \mathrm{H}), 5.07(\mathrm{dt}, J=50.8,3.9 \mathrm{~Hz}, 1 \mathrm{H}), 4.78-4.67(\mathrm{~m}, 1 \mathrm{H}), 4.17-4.05(\mathrm{~m}, 1 \mathrm{H}), 3.99-3.81(\mathrm{~m}, 1 \mathrm{H})$, $2.44(\mathrm{br}, 1 \mathrm{H}), 1.52(\mathrm{~s}, 9 \mathrm{H}) .{ }^{19} \mathrm{~F}-\mathrm{NMR}\left(376 \mathrm{MHz}, \mathrm{CDCl}_{3}\right) \delta-200.57 .{ }^{13} \mathrm{C}-\mathrm{NMR}(101 \mathrm{MHz}$, Chloroform- $d$ ) $\delta 153.5,135.2,129.6,128.2,126.9,124.8,89.4\left(\mathrm{C}-\mathrm{F},{ }^{1} J_{\mathrm{CF}}=176.8 \mathrm{~Hz}\right), 87.7$ $\left(\mathrm{C}-\mathrm{F},{ }^{1} J_{\mathrm{CF}}=176.8 \mathrm{~Hz}\right), 82.3,67.7\left(\mathrm{C}-\mathrm{F},{ }^{2} J_{\mathrm{CF}}=20.2 \mathrm{~Hz}\right), 67.5\left(\mathrm{C}-\mathrm{F},{ }^{2} J_{\mathrm{CF}}=20.2 \mathrm{~Hz}\right), 46.7(\mathrm{C}-$ $\left.\mathrm{F}^{2} J_{\mathrm{CF}}=23.2 \mathrm{~Hz}\right), 46.5\left(\mathrm{C}-\mathrm{F},{ }^{2} J_{\mathrm{CF}}=23.2 \mathrm{~Hz}\right), 28.4$. HRMS (APCI): $m / z[\mathrm{M}+\mathrm{H}]^{+}$calcd. for $\mathrm{C}_{14} \mathrm{H}_{18} \mathrm{ClFNO}_{3}$ 302.0954, found 302.0955. SFC: Chiralpak IE, scCO $\mathrm{CO}_{2} / \mathrm{MeOH} 94 / 6,2.0$ $\mathrm{mL} / \mathrm{min}, \mathrm{P}=100$ bar, $\lambda=254 \mathrm{~nm}, \mathrm{t}_{\mathrm{R}}[$ trans $]=6.95 \mathrm{~min}, \mathrm{t}_{\mathrm{R}}[$ trans $]=7.49 \mathrm{~min}, \mathrm{t}_{\mathrm{R}}$ [cis$(3 S, 4 R)]=10.31 \mathrm{~min}, \mathrm{t}_{\mathrm{R}}[$ cis- $(3 R, 4 \mathrm{~S})]=11.09 \mathrm{~min}$ (major).

tert-Butyl (3R,4S)-6-bromo-3-fluoro-4-hydroxy-3,4-dihydroquinoline-1(2H)-carboxylate (3i). $80 \mathrm{mg}$ of tert-butyl 3-fluoro-6-bromo-4-oxo-3,4-dihydroquinoline-1(2H)-carboxylate (2i) $(0.24 \mathrm{mmol} ; 1.0$ equiv) were used according to the described procedure (reaction time of $3 \mathrm{~h}$ ). The product was purified by flash column chromatography on silica gel (petroleum ether/ethyl acetate 75:25) and yielded $97 \mathrm{mg}$ of a beige/white solid (95\%). m.p. $128-131{ }^{\circ} \mathrm{C}$ $\mathrm{dr}($ cis/trans $)=98: 2, \mathrm{ee}_{\text {cis }}=>99 \% . \quad[\alpha]_{\mathrm{D}}{ }^{22}=-15.6\left(\right.$ c 1.0, $\left.\mathrm{CHCl}_{3}\right) .{ }^{1} \mathrm{H}-\mathrm{NMR}(400 \mathrm{MHz}$, Chloroform- $d$ ) $\delta 7.68-7.65(\mathrm{~m}, 1 \mathrm{H}), 7.59(\mathrm{~d}, J=8.9 \mathrm{~Hz}, 1 \mathrm{H}), 7.37(\mathrm{dd}, J=8.9,2.4 \mathrm{~Hz}, 1 \mathrm{H})$, $5.05(\mathrm{dq}, J=51.0,3.6 \mathrm{~Hz}, 1 \mathrm{H}), 4.71(\mathrm{ddd}, J=23.2,9.3,3.1 \mathrm{~Hz}, 1 \mathrm{H}), 4.10(\mathrm{ddd}, J=15.7,14.4$, $4.1 \mathrm{~Hz}, 1 \mathrm{H}), 3.88(\mathrm{ddd}, J=33.1,14.5,3.5 \mathrm{~Hz}, 1 \mathrm{H}), 2.52(\mathrm{~d}, J=9.4 \mathrm{~Hz}, 1 \mathrm{H}), 1.52(\mathrm{~s}, 9 \mathrm{H})$. ${ }^{19} \mathrm{~F}-\mathrm{NMR}\left(376 \mathrm{MHz}\right.$, Chloroform-d) $\delta-200.49 .{ }^{13} \mathrm{C}-\mathrm{NMR}(101 \mathrm{MHz}$, Chloroform- $d$ ) $\delta 153.5$, $135.7,131.1,130.0,129.9,125.1,117.2,89.4\left(\mathrm{C}-\mathrm{F},{ }^{1} J_{\mathrm{CF}}=176.8 \mathrm{~Hz}\right), 87.6\left(\mathrm{C}-\mathrm{F},{ }^{1} J_{\mathrm{CF}}=176.8 \mathrm{~Hz}\right)$, 82.3, $67.7\left(\mathrm{C}-\mathrm{F},{ }^{2} J_{\mathrm{CF}}=20.2 \mathrm{~Hz}\right), 67.5\left(\mathrm{C}-\mathrm{F},{ }^{2} J_{\mathrm{CF}}=20.2 \mathrm{~Hz}\right), 46.7\left(\mathrm{C}-\mathrm{F},{ }^{2} J_{\mathrm{CF}}=23.2 \mathrm{~Hz}\right), 46.4$ $\left(\mathrm{C}-\mathrm{F},{ }^{2} J_{\mathrm{CF}}=23.2 \mathrm{~Hz}\right), 28.4$. HRMS (APCI): $\mathrm{m} / z\left[\mathrm{M}+\mathrm{NH}_{4}\right]^{+}$calcd. for $\mathrm{C}_{14} \mathrm{H}_{21} \mathrm{BrFN}_{2} \mathrm{O}_{3}$ 363.0714, found 363.0714. SFC: Chiralpak IE, $s c \mathrm{CO}_{2} / \mathrm{MeOH} 93 / 7,2.0 \mathrm{~mL} / \mathrm{min}, \mathrm{P}=100 \mathrm{bar}$, $\lambda=254 \mathrm{~nm}, \mathrm{t}_{\mathrm{R}}[$ trans $]=7.72 \mathrm{~min}, \mathrm{t}_{\mathrm{R}}[$ cis- $(3 S, 4 R)]=11.07 \mathrm{~min}, \mathrm{t}_{\mathrm{R}}[$ cis- $(3 R, 4 \mathrm{~S})]=11.96 \mathrm{~min}$ (major).

tert-Butyl (3R,4S)-3-fluoro-4-hydroxy-6-iodo-3,4-dihydroquinoline-1(2H)-carboxylate (3j). $80 \mathrm{mg}$ of tert-butyl 3-fluoro-6-iodo-4-oxo-3,4-dihydroquinoline-1(2H)-carboxylate $(2 \mathbf{j})$ $(0.20 \mathrm{mmol} ; 1.0$ equiv) were used according to the described procedure (reaction time of $3 \mathrm{~h}$ ). The product was purified by flash column chromatography on silica gel (petroleum ether/ethyl acetate 75:25) and yielded $97 \mathrm{mg}$ of a beige/white solid (94\%). m.p. $125-128^{\circ} \mathrm{C}$ $\mathrm{dr}($ cis/trans $)=98: 2, \mathrm{ee}_{\text {cis }}=>99 \% .[\alpha]_{\mathrm{D}}{ }^{22}=-19.6\left(c 1.0, \mathrm{CHCl}_{3}\right) .{ }^{1} \mathrm{H}-\mathrm{NMR}(400 \mathrm{MHz}$, Chloroform- $d) \delta 7.84(\mathrm{~d}, J=0.8 \mathrm{~Hz}, 1 \mathrm{H}), 7.56(\mathrm{dt}, J=8.7,1.6 \mathrm{~Hz}, 1 \mathrm{H}), 7.47(\mathrm{~d}, J=8.8 \mathrm{~Hz}$, $1 \mathrm{H}), 5.04(\mathrm{dt}, J=50.9,3.7 \mathrm{~Hz}, 1 \mathrm{H}), 4.71(\mathrm{dd}, J=23.3,8.8 \mathrm{~Hz}, 1 \mathrm{H}), 4.10(\mathrm{ddt}, J=18.6,15.1$, 3.6 Hz, 1H), 3.97-3.78 (m, 1H), $2.46(\mathrm{~s}, 1 \mathrm{H}), 1.52(\mathrm{~s}, 9 \mathrm{H}) .{ }^{19} \mathrm{~F}-\mathrm{NMR}(376 \mathrm{MHz}$, Chloroform- $d$ ) $\delta$-200.57. ${ }^{13} \mathrm{C}-\mathrm{NMR}(101 \mathrm{MHz}$, Chloroform-d) $\delta 153.4,137.0,136.6,135.9,130.1,125.3$, $89.3\left(\mathrm{C}-\mathrm{F},{ }^{1} J_{\mathrm{CF}}=176.8 \mathrm{~Hz}\right), 87.8\left(\mathrm{C}-\mathrm{F},{ }^{1} J_{\mathrm{CF}}=176.8 \mathrm{~Hz}\right), 87.5,82.4,67.5\left(\mathrm{C}-\mathrm{F},{ }^{2} J_{\mathrm{CF}}=20.2 \mathrm{~Hz}\right)$, $67.3\left(\mathrm{C}-\mathrm{F},{ }^{2} J_{\mathrm{CF}}=20.2 \mathrm{~Hz}\right), 46.6\left(\mathrm{C}-\mathrm{F},{ }^{2} J_{\mathrm{CF}}=23.2 \mathrm{~Hz}\right), 46.3\left(\mathrm{C}-\mathrm{F},{ }^{2} J_{\mathrm{CF}}=23.2 \mathrm{~Hz}\right), 28.4$. HRMS (APCI): $m / z$ [M $\left.+\mathrm{NH}_{4}\right]^{+}$calcd. for $\mathrm{C}_{14} \mathrm{H}_{21} \mathrm{FIN}_{2} \mathrm{O}_{3} 411.0575$, found 411.0577. SFC: Chiralpak $\mathrm{IE}, \mathrm{scCO}_{2} / \mathrm{MeOH} 93 / 7,2.0 \mathrm{~mL} / \mathrm{min}, \mathrm{P}=100 \mathrm{bar}, \lambda=254 \mathrm{~nm}, \mathrm{t}_{\mathrm{R}}[$ trans $]=10.92 \mathrm{~min}, \mathrm{t}_{\mathrm{R}}$ $[$ trans $]=11.41 \mathrm{~min}, \mathrm{t}_{\mathrm{R}}[$ cis- $(3 S, 4 R)]=15.73 \mathrm{~min}, \mathrm{t}_{\mathrm{R}}[$ cis- $(3 R, 4 \mathrm{~S})]=17.25 \mathrm{~min}$ (major). 


\subsection{Gram-Scale Experiment}

In a $30 \mathrm{~mL}$-round-bottom tube charged with tert-butyl 3-fluoro-4-oxo-3,4-dihydroquinoline- $1(2 \mathrm{H})$-carboxylate $(2 \mathrm{a}, 1.0 \mathrm{~g}, 3.8 \mathrm{mmol} ; 1.0$ equiv) and the catalyst $(R, R)-\mathrm{D}(12.4 \mathrm{mg}$, $12 \mu \mathrm{mol} ; 0.005$ equiv) set under argon were added $10 \mathrm{~mL}$ of acetonitrile. The mixture was stirred for one minute before adding by syringe a (1:1) mixture of formic acid and triethylamine $\left(3.40 \mathrm{~mL}, 16 \mathrm{mmol} ; 6.0\right.$ equiv). The reaction mixture was stirred at $40{ }^{\circ} \mathrm{C}$ for $24 \mathrm{~h}$. Then it was cooled down and quenched with $30 \mathrm{~mL}$ of $\mathrm{NaHCO}_{3}$ aqueous solution. The mixture was extracted with $\mathrm{CH}_{2} \mathrm{Cl}_{2}(2 \times 40 \mathrm{~mL})$ and the organic layers dried over $\mathrm{MgSO}_{4}$, filtered, and concentrated under vacuum. The diastereoisomeric ratio was determined by ${ }^{1} \mathrm{H}-\mathrm{NMR}$ analysis of the crude product. The product was purified with a flash column chromatography on silica gel (petroleum ether/EtOAc 70:30). $960 \mathrm{mg}$ (96\%) of (3R,4R)3-fluorochroman-4-ol (3a) was obtained as a white solid. dr (cis/trans) 97:3, ee cis $=>99 \%$. ${ }^{1} \mathrm{H}-\mathrm{NMR}(400 \mathrm{MHz}$, Chloroform- $d) \delta 7.70(\mathrm{~d}, J=8.3 \mathrm{~Hz}, 1 \mathrm{H}), 7.51(\mathrm{dt}, J=7.6,1.4 \mathrm{~Hz}, 1 \mathrm{H})$, $7.34-7.24(\mathrm{~m}, 1 \mathrm{H}), 7.14(\mathrm{td}, J=7.5,1.2 \mathrm{~Hz}, 1 \mathrm{H}), 5.05(\mathrm{ddt}, J=50.8,4.8,3.5 \mathrm{~Hz}, 1 \mathrm{H}), 4.78(\mathrm{dd}$, $J=21.5,5.9 \mathrm{~Hz}, 1 \mathrm{H}), 4.10(\mathrm{ddd}, J=14.9,14.2,4.8 \mathrm{~Hz}, 1 \mathrm{H}), 3.94(\mathrm{ddd}, J=29.9,14.2,3.7 \mathrm{~Hz}$, 1H), $2.38(\mathrm{~d}, J=8.3 \mathrm{~Hz}, 1 \mathrm{H}), 1.53(\mathrm{~s}, 9 \mathrm{H}) .{ }^{19} \mathrm{~F}-\mathrm{NMR}$ (376 MHz, Chloroform-d) $\delta-200.34$. ${ }^{13} \mathrm{C}-\mathrm{NMR}(101 \mathrm{MHz}$, Chloroform-d) $\delta$ 153.8, 136.8, 128.2, 127.9, 127.3, 124.3, 123.5, 89.6 $\left(\mathrm{C}-\mathrm{F},{ }^{1} J_{\mathrm{CF}}=176.8 \mathrm{~Hz}\right), 87.9\left(\mathrm{C}-\mathrm{F},{ }^{1} J_{\mathrm{CF}}=176.8 \mathrm{~Hz}\right), 81.9,68.0\left(\mathrm{C}-\mathrm{F},{ }^{2} J_{\mathrm{CF}}=19.2 \mathrm{~Hz}\right), 67.8(\mathrm{C}-\mathrm{F}$, $\left.{ }^{2} J_{\mathrm{CF}}=19.2 \mathrm{~Hz}\right), 46.3\left(\mathrm{C}-\mathrm{F},{ }^{2} J_{\mathrm{CF}}=23.2 \mathrm{~Hz}\right), 46.1\left(\mathrm{C}-\mathrm{F},{ }^{2} J_{\mathrm{CF}}=23.2 \mathrm{~Hz}\right), 28.4$. SFC: Chiralpak $\mathrm{IE}, \mathrm{scCO}_{2} / \mathrm{EtOH} 96 / 4,2.0 \mathrm{~mL} / \mathrm{min}, \mathrm{P}=100 \mathrm{bar}, \lambda=215 \mathrm{~nm}, \mathrm{t}_{\mathrm{R}}[$ trans $]=11.04 \mathrm{~min}, \mathrm{t}_{\mathrm{R}}$ $[$ trans $]=12.85 \mathrm{~min}, \mathrm{t}_{\mathrm{R}}[$ cis- $(3 R, 4 \mathrm{~S})]=14.54 \mathrm{~min}($ major $), \mathrm{t}_{\mathrm{R}}[$ cis- $(3 S, 4 R)]=15.43 \mathrm{~min}$.

\subsection{Sonogashira Coupling Reaction}

Tert-Butyl (3R,4S)-3-fluoro-4-hydroxy-6-(phenylethynyl)-3,4-dihydroquinoline-1(2H)carboxylate (4). Tert-Butyl (3R,4S)-3-fluoro-4-hydroxy-6-iodo-3,4-dihydroquinoline-1(2H)carboxylate (3j) (77 mg; $0.2 \mathrm{mmol} ; 1.0$ equiv) was introduced into a $10 \mathrm{~mL}$ round-bottom tube at open air with $1 \mathrm{~mL}$ of a $1: 1 \mathrm{CH}_{3} \mathrm{CN} / \mathrm{Et}_{3} \mathrm{~N}$ mixture. Next, phenylacetylene was added (31 $\mu \mathrm{L} ; 0.3 \mathrm{mmol} ; 1.5$ equiv) followed by CuI (2.8 mg; $10 \mu \mathrm{mol} ; 0.05$ equiv) and $\mathrm{PdCl}_{2}\left(\mathrm{PPh}_{3}\right)_{2}(6.4 \mathrm{mg} ; 6 \mu \mathrm{mol} ; 0.03$ equiv). The mixture was stirred for $30 \mathrm{~min}$ at room temperature. It was then quenched with $2 \mathrm{~mL}$ of $\mathrm{NH}_{4} \mathrm{Cl}$ saturated solution and extracted with EtOAc $(3 \times 10 \mathrm{~mL})$. The combined organic phases were dried with $\mathrm{MgSO}_{4}$, filtered, and concentrated under reduced pressure. The crude product was purified by flash column chromatography on silica gel, (petroleum ether/EtOAc/toluene 7:2:1) to afford 4 as a pale-yellow solid (49 mg; 68\%). ${ }^{1} \mathrm{H}-\mathrm{NMR}\left(400 \mathrm{MHz}, \mathrm{CDCl}_{3}\right) \delta 7.76-7.69(\mathrm{~m}, 2 \mathrm{H}), 7.55-7.49$ $(\mathrm{m}, 2 \mathrm{H}), 7.44(\mathrm{ddd}, J=8.6,2.1,0.6 \mathrm{~Hz}, 1 \mathrm{H}), 7.37-7.30(\mathrm{~m}, 3 \mathrm{H}), 5.06(\mathrm{ddt}, J=50.8,4.5,3.4 \mathrm{~Hz}$, $1 \mathrm{H}), 4.76(\mathrm{dd}, J=23.3,6.0 \mathrm{~Hz}, 1 \mathrm{H}), 4.13(\mathrm{ddd}, J=15.3,14.3,4.4 \mathrm{~Hz}, 1 \mathrm{H}), 3.91(\mathrm{ddd}, J=32.0$, 14.3, $3.5 \mathrm{~Hz}, 1 \mathrm{H}), 2.52(\mathrm{~d}, J=9.0 \mathrm{~Hz}, 1 \mathrm{H}), 1.54(\mathrm{~s}, 9 \mathrm{H}) .{ }^{19} \mathrm{~F}-\mathrm{NMR}\left(376 \mathrm{MHz}, \mathrm{CDCl}_{3}\right) \delta$ -200.41. ${ }^{13} \mathrm{C}-\mathrm{NMR}\left(101 \mathrm{MHz}, \mathrm{CDCl}_{3}\right) \delta 153.5,136.7,131.7,131.3,130.5,128.5,128.3,127.9$, $123.4,123.2,118.9,89.4,89.3\left(\mathrm{C}-\mathrm{F},{ }^{1} J_{\mathrm{CF}}=176.8 \mathrm{~Hz}\right), 89.1,87.6\left(\mathrm{C}-\mathrm{F},{ }^{1} J_{\mathrm{CF}}=176.8 \mathrm{~Hz}\right), 82.3$, $67.7\left(\mathrm{C}-\mathrm{F},{ }^{2} J_{\mathrm{CF}}=20.2 \mathrm{~Hz}\right), 67.5\left(\mathrm{C}-\mathrm{F},{ }^{2} J_{\mathrm{CF}}=20.2 \mathrm{~Hz}\right), 46.6\left(\mathrm{C}-\mathrm{F},{ }^{2} J_{\mathrm{CF}}=23.2 \mathrm{~Hz}\right), 46.4(\mathrm{C}-\mathrm{F}$, $\left.{ }^{2} J_{\mathrm{CF}}=23.2 \mathrm{~Hz}\right), 28.4$. MS (ESI) $[\mathrm{M}-\mathrm{Boc}+\mathrm{H}]^{+}=267$.

\subsection{N-Boc Deprotection}

(3R,4S)-3-Fluoro-1,2,3,4-tetrahydroquinolin-4-ol (5). Tert-Butyl (3R,4S)-3-fluoro-4hydroxy-3,4-dihydroquinoline- $1(2 \mathrm{H})$-carboxylate $3 \mathrm{a}(200 \mathrm{mg} ; 0.75 \mathrm{mmol} ; 1.0$ equiv) was introduced in a $50 \mathrm{~mL}$ round-bottom tube set under argon. $3.0 \mathrm{~mL}$ of 1,4-dioxane were added followed by $5.0 \mathrm{~mL}$ of HPLC-grade $\mathrm{H}_{2} \mathrm{O}$. The mixture was heated to reflux for $34 \mathrm{~h}$ [26]. It was then allowed to cool down to room temperature, and it was extracted with MTBE $(3 \times 20 \mathrm{~mL})$. The combined organic phases were dried with $\mathrm{MgSO}_{4}$, filtered, and concentrated under reduced pressure. The crude product was purified by flash column chromatography on silica gel (petroleum ether/EtOAc 8:2 to 7:3) to afford 5 as a pale-yellow solid (112 mg; 90\%). ${ }^{1} \mathrm{H}-\mathrm{NMR}\left(400 \mathrm{MHz}, \mathrm{CDCl}_{3}\right) \delta 7.35(\mathrm{dt}, J=7.8,1.0 \mathrm{~Hz}, 1 \mathrm{H}), 7.11$ (ddd, $J=8.5,7.5,1.6 \mathrm{~Hz}, 1 \mathrm{H}), 6.75(\mathrm{td}, J=7.4,1.2 \mathrm{~Hz}, 1 \mathrm{H}), 6.55(\mathrm{dd}, J=8.0,1.1 \mathrm{~Hz}, 1 \mathrm{H}), 5.08-4.75$ $(\mathrm{m}, 2 \mathrm{H}), 3.87(\mathrm{~s}, 1 \mathrm{H}), 3.66(\mathrm{dt}, J=11.9,7.9 \mathrm{~Hz}, 1 \mathrm{H}), 3.45(\mathrm{dddd}, J=22.2,11.9,3.3,1.4 \mathrm{~Hz}$, 
1H), 2.26 (s, 1H). ${ }^{19} \mathrm{~F}-\mathrm{NMR}\left(376 \mathrm{MHz}, \mathrm{CDCl}_{3}\right) \delta-203.21 .{ }^{13} \mathrm{C}-\mathrm{NMR}\left(101 \mathrm{MHz}, \mathrm{CDCl}_{3}\right) \delta$ $143.4,129.7,129.6,120.0,118.2,114.2,88.8\left(\mathrm{C}-\mathrm{F},{ }^{1} J_{\mathrm{CF}}=176.8 \mathrm{~Hz}\right), 87.1\left(\mathrm{C}-\mathrm{F},{ }^{1} J_{\mathrm{CF}}=176.8 \mathrm{~Hz}\right)$, $67.3\left(\mathrm{C}-\mathrm{F},{ }^{2} J_{\mathrm{CF}}=19.2 \mathrm{~Hz}\right), 67.1\left(\mathrm{C}-\mathrm{F},{ }^{2} J_{\mathrm{CF}}=19.2 \mathrm{~Hz}\right), 41.8\left(\mathrm{C}-\mathrm{F},{ }^{2} J_{\mathrm{CF}}=24.2 \mathrm{~Hz}\right), 41.6(\mathrm{C}-\mathrm{F}$, ${ }^{2} J_{\mathrm{CF}}=24.2 \mathrm{~Hz}$ ). HRMS (ESI) $\mathrm{m} / z$ : $[\mathrm{M}+\mathrm{H}]^{+}$calcd. for $\mathrm{C}_{9} \mathrm{H}_{11} \mathrm{FNO} 168.0819$. Found 168.0819.

\section{Conclusions}

In summary, a straightforward method to access enantiomerically and diastereomerically enriched cis-fluoro-dihydrotetrahydroquinolinols has been developed. Ru catalyst $(R, R)$-TsDENEB ensures an efficient asymmetric transfer hydrogenation/dynamic kinetic resolution (ATH/DKR) under mild conditions (acetonitrile as solvent and heating at $40{ }^{\circ} \mathrm{C}$ ) and low catalyst loading $(0.5 \mathrm{~mol} \%)$. The reaction was performed successfully on a series of 3-fluoro-dihydrotetrahydroquinolin-4-ones substituted with several electron-donating or electron-withdrawing groups with excellent yields and diastereo- and enantioselectivities.

Supplementary Materials: A supporting information including NMR and SFC spectra, is available online.

Author Contributions: Conceptualization, P.P. and V.R.-V.; methodology, R.M.B.; supervision, P.P. and V.R.-V.; writing—original draft preparation, R.M.B.; writing—review and editing, P.P. and V.R.-V. All authors have read and agreed to the published version of the manuscript.

Funding: This work was supported by the Ministère de l'Enseignement Supérieur de la Recherche et de l'Innovation (MESRI), grant number 3430/2019 and the Centre National de la Recherche Scientifique (CNRS).

Institutional Review Board Statement: Not applicable.

Informed Consent Statement: Not applicable.

Data Availability Statement: CCDC 2132626 contains the supplementary crystallographic data for this paper. These data can be obtained free of charge via www.ccdc.cam.ac.uk/data_request/cif (accessed on 5 January 2022), or by emailing data_request@ccdc.cam.ac.uk, or by contacting The Cambridge Crystallographic Data Centre, 12 Union Road, Cambridge CB2 1EZ, UK; Fax: +44-1223336033.

Acknowledgments: We gratefully acknowledge the MESRI for a grant to R.M.B. We thank J. Forté (Sorbonne Université, Paris) for solving the X-ray structure of compound 2a. Dedicated to H. B. Kagan for his outstanding contribution to asymmetric catalysis and organometallic chemistry.

Conflicts of Interest: The authors declare no conflict of interest.

Sample Availability: Samples of the compounds are not available from the authors.

\section{References}

1. Wang, J.; Sánchez-Roselló, M.; Aceña, J.L.; Del Pozo, C.; Sorochinsky, A.E.; Fustero, S.; Soloshonok, V.A.; Liu, H. Fluorine in Pharmaceutical Industry: Fluorine-Containing Drugs Introduced to the Market in the Last Decade (2001-2011). Chem. Rev. 2014, 114, 2432. [CrossRef]

2. Mei, H.; Han, J.; Fustero, S.; Medio-Simon, M.; Sedgwick, D.M.; Santi, C.; Ruzziconi, R.; Soloshonok, V.A. Fluorine-Containing Drugs Approved by the FDA in 2018. Chem. Eur. J. 2019, 25, 11797. [CrossRef]

3. Caron, S. Where Does the Fluorine Come From? A Review on the Challenges Associated with the Synthesis of Organofluorine Compounds. Org. Process Res. Dev. 2020, 24, 470. [CrossRef]

4. Sridharan, V.; Suryavanshi, P.A.; Menéndez, J.C. Advances in the Chemistry of Tetrahydroquinolines. Chem. Rev. 2011, 111, 7157. [CrossRef]

5. Ros, A.; Magriz, A.; Dietrich, H.; Fernández, R.; Alvarez, E.; Lassaletta, J.M. Enantioselective Synthesis of Vicinal Halohydrins via Dynamic Kinetic Resolution. Org. Lett. 2006, 8, 127. [CrossRef] [PubMed]

6. Ayad, T.; Phansavath, P.; Ratovelomanana-Vidal, V. Transition Metal-Catalyzed Asymmetric Hydrogenation and Transfer Hydrogenation: Sustainable Chemistry to Access Bioactive Molecules. Chem. Rec. 2016, 16, 2754. [CrossRef] [PubMed]

7. Zheng, L.-S.; Phansavath, P.; Ratovelomanana-Vidal, V. Ruthenium-Catalyzed Dynamic Kinetic Asymmetric Transfer Hydrogenation: Stereoselective Access to Syn 2-(1,2,3,4-Tetrahydro-1-Isoquinolyl)Ethanol Derivatives. Org. Chem. Front. $2018,5,1366$. [CrossRef] 
8. Zheng, L.-S.; Férard, C.; Phansavath, P.; Ratovelomanana-Vidal, V. Rhodium-Mediated Asymmetric Transfer Hydrogenation: A Diastereo- and Enantioselective Synthesis of Syn- $\alpha$-Amido $\beta$-Hydroxy Esters. Chem. Commun. 2018, 54, 283. [CrossRef]

9. Zheng, L.-S.; Phansavath, P.; Ratovelomanana-Vidal, V. Synthesis of Enantioenriched $\alpha, \alpha$-Dichloro- and $\alpha, \alpha$-Difluoro- $\beta$-Hydroxy Esters and Amides by Ruthenium-Catalyzed Asymmetric Transfer Hydrogenation. Org. Lett. 2018, 20, 5107. [CrossRef]

10. He, B.; Phansavath, P.; Ratovelomanana-Vidal, V. Rh-Mediated Asymmetric-Transfer Hydrogenation of 3-Substituted Chromones: A Route to Enantioenriched cis-3-(Hydroxymethyl)chroman-4-ol Derivatives through Dynamic Kinetic Resolution. Org. Lett. 2019, 21, 3276. [CrossRef]

11. He, B.; Phansavath, P.; Ratovelomanana-Vidal, V. Rhodium-Catalyzed Asymmetric Transfer Hydrogenation of 4-Quinolone Derivatives. Org. Chem. Front. 2020, 7, 975. [CrossRef]

12. Westermeyer, A.; Guillamot, G.; Phansavath, P.; Ratovelomanana-Vidal, V. Synthesis of Enantioenriched $\beta$-Hydroxy- $\gamma$-acetal Enamides by Rhodium-catalyzed Asymmetric Transfer Hydrogenation. Org. Lett. 2020, 22, 3911. [CrossRef] [PubMed]

13. Molina Betancourt, R.; Phansavath, P.; Ratovelomanana-Vidal, V. Rhodium-Catalyzed Asymmetric Transfer Hydrogenation/Dynamic Kinetic Resolution of 3-Benzylidene-Chromanones. Org. Lett. 2021, 23, 1621. [CrossRef]

14. Molina Betancourt, R.; Phansavath, P.; Ratovelomanana-Vidal, V. Ru(II)-Catalyzed Asymmetric Transfer Hydrogenation of 3-Fluorochromanone Derivatives to Access Enantioenriched Cis-3-Fluorochroman-4-Ols through Dynamic Kinetic Resolution. J. Org. Chem. 2021, 86, 12054. [CrossRef] [PubMed]

15. Noyori, R.; Tokunaga, M.; Kitamura, M. Stereoselective Organic Synthesis via Dynamic Kinetic Resolution. Bull. Chem. Soc. Jpn. 1995, 68, 36. [CrossRef]

16. Pellissier, H. Recent Developments in Dynamic Kinetic Resolution. Tetrahedron 2011, 67, 3769. [CrossRef]

17. Samec, J.S.M.; Bäckvall, J.-E.; Andersson, P.G.; Brandt, P. Mechanistic Aspects of Transition Metal-Catalyzed Hydrogen Transfer Reactions. Chem. Soc. Rev. 2006, 35, 237. [CrossRef]

18. Blacker, A.J.; de Vries, J.G. Handbook of Homogeneous Hydrogenation; Elsevier, C.J., Ed.; Wiley-VCH: Weinheim, German, 2007.

19. Foubelo, F.; Nájera, C.; Yus, M. Catalytic Asymmetric Transfer Hydrogenation of Ketones: Recent Advances. Tetrahedron: Asymmetry 2015, 26, 769. [CrossRef]

20. Echeverria, P.-G.; Ayad, T.; Phansavath, P.; Ratovelomanana-Vidal, V. Recent Developments in Asymmetric Hydrogenation and Transfer Hydrogenation of Ketones and Imines through Dynamic Kinetic Resolution. Synthesis 2016, 48, 2523. [CrossRef]

21. Matsunami, A.; Kayaki, Y. Upgrading and Expanding the Scope of Homogeneous Transfer Hydrogenation. Tetrahedron Lett. 2018, 59, 504. [CrossRef]

22. Talavera, G.; Santana Fariña, A.; Zanotti-Gerosa, A.; Nedden, H.G. Structural Diversity in Ruthenium-Catalyzed Asymmetric Transfer Hydrogenation Reactions. In Topics in Organometallic Chemistry; Springer: Berlin, Heidelberg, German, 2019.

23. Molina Betancourt, R.; Echeverria, P.-G.; Ayad, T.; Phansavath, P.; Ratovelomanana-Vidal, V. Recent Progress and Applications of Transition-Metal-Catalyzed Asymmetric Hydrogenation and Transfer Hydrogenation of Ketones and Imines through Dynamic Kinetic Resolution. Synthesis 2021, 53, 30.

24. Cotman, A.E. Escaping from Flatland: Stereoconvergent Synthesis of Three-Dimensional Scaffolds via Ruthenium(II)-Catalyzed Noyori-Ikariya Transfer Hydrogenation. Chem. Eur. J. 2021, 27, 39. [CrossRef] [PubMed]

25. Bélanger, E.; Houzé, C.; Guimond, N.; Cantin, K.; Paquin, J.-F. Unexpected Effect of the Fluorine Atom on the Optimal Ligand-toPalladium Ratio in the Enantioselective Pd-Catalyzed Allylation Reaction of Fluorinated Enol Carbonates. Chem. Commun. 2008, 28, 3251. [CrossRef] [PubMed]

26. Wang, J.; Liang, Y.-L.; Qu, J. Boiling water-catalyzed neutral and selective N-Boc deprotection. Chem. Commun. 2009, 34, 5144. [CrossRef] 